\title{
The electronic proprieties of the silver clusters
}

\author{
Mariana Virginia Popa ${ }^{1,2}$ \\ ${ }^{1}$ Electronic and Telecomunication, Autonomous University of the Hidalgo State, Mexico \\ ${ }^{2}$ Regularización 2000, C/Dr. Agustin Torres Cravioto 102B, col. Doctores, C. P. 42090, Pachuca, Hidalgo, México
}

\section{Email address:}

virginia_popa@yahoo.com.mx,pmarianavirginia@yahoo.com

\section{To cite this article:}

Mariana Virginia Popa. The Electronic Proprieties of the Silver Clusters. International Journal of Computational and Theoretical Chemistry. Vol. 2, No. 6, 2014, pp. 46-68. doi: 10.11648/j.ijctc.20140206.11

\begin{abstract}
En this article are presented the theorics work for clarify the structure of all silver cluster and are compareted the results with experimental data for see which levels of theory describe better the propriety of the silver cluster. For $\mathrm{Ag}_{2}-\mathrm{A}_{5}$ are reach different value of the bond, ionization potentials and frequencies, electron affinities and biding energy method employed $\mathrm{ab}$ initio and relativystic bases. Are optimization with the following levels of theorie: HF/LANL1MB, HF/LANL2MB, HF/LANL2DZ, B3LYP/LANL1MB, B3LYP/LANL2MB， B3LYP/LANL2DZ， MP2/LANL2DZ, DFT/PBE/SDD and $\mathrm{DFT} / \mathrm{PBE} / 3-21 \mathrm{G}^{* *}$.
\end{abstract}

Keywords: Silver, Relativystic Effects, Metal Clusters

\section{Introduction}

In the past two decade has been showing great interest for explanation the electronics proprieties of silver cluster with the nanometre dimension due to the proprieties who to exhibit the cluster generally, are diferent of the solid state $[1,2,3]$ and molecular [4].

The study of the structure of the clusters of dimension nano to require to compare the date of the diferents experiments and theorie.

The extraordinary proprieties of silver clusters to give a reason a much investigator to realice the worcks in photography, catalisis and news materials of electronic $[5,6-$ 14].

Has been moderate that the relativistic effects influe in the electronic proprieties, so how in the geometry of the metals of transition so that gold, cooper and silver [15]. The orbital $6 \mathrm{~s}$ in gold to contract for the relativistic effect [15].

In [16] has been make the studie with ion of silver with espectroscopy of mass. The neutral clusters has been obtained bombardment sheet of metal with ion Xe. Has been regarde who the distribution of masa similar for negative and positive clusters. Has been analyze the structure of anion cluster $\operatorname{Ag}_{n}(n=1-9)$ useful the electronic espectrofotometry with gas He [17]. With nuclear magnetic espectroscopy of resonance and ultraviolet absorcion espectroscopy has been evidence the Jahn-Teller effect for $\mathrm{Ag}_{3}[18,19]$. Has been employed optic espectroscopy in the Ar matrix to characterize $\mathrm{Ag}_{4}$ and has been evidence two isomerous stables $\mathrm{D}_{2 \mathrm{~h}}$ and $\mathrm{C}_{2 \mathrm{~V}}$ with the difference between isomerous $0 \mathrm{f}$ $0.2 \mathrm{eV}$ [20]. Employed the electronic spin resonance has been evidence the dimer, trimeros and pentamer with the JahnTeller effect for the silver clusters [21, 22].

With the generator of $\mathrm{N}_{2}$ laser, the vertical ionization potentials for $\operatorname{Ag}_{n}(n<100)$ has been study $[23,24]$.

The fotoelectronic espectroscopy $[25,26]$ is a powerful technical for to study of electronic structure of metalic clusters and an very good method for adiabatic electronic affinity. The anionic clusters are separated with an massa espectromettre.

Has been evidence the alternate of the values for vertical electronic affinity with the variation even/odd (until 40 atoms) of silver used the fotoelectronic spectra in UV with He gas and laser with energy of the photon $6.4 \mathrm{y} 7.9 \mathrm{eV}[27,28]$.

The frequencyof the ${ }^{2} \mathrm{~B}_{2}$ and ${ }^{2} \mathrm{~A}_{1}$ has been evidence employed the optic espectra emision with $\mathrm{Ag}_{3}$ evaporation at low pressure in He (100Torr) [29].

With the fotoelectronic espectra has been evidence the frequencies of the silver cluster $\leq 70$ atoms [30]. Employed the optic resonancie absorption for $\mathrm{Ag}_{4}^{+}$has been evidence the fotofragmentation of anion cluster [31].

In the literature to attain various works with Ag clusters $[32,40]$.

\section{Results and Discussion}

To introduce the electronic effects of correlation with B3LYP and B3PW91 functionals and relativistic effect with 
LANL2DZ and LANL2MB bases for $\mathrm{Ag}_{2}$ are report the studys in reference [36], with BPW91 and SVWN IIa LANL2DZ base, the mistake for geometry are 3-4\% y for frecuencies $6-8 \%$. Also are reach the difference of the proprieties of $\mathrm{Ag}_{2}$ above mentionen, with MP2 and $\mathrm{S}$ VWN+Beke-Perdew functionals [37]. To be able that $\mathrm{Ag}_{2}$ the distances are satisfy compared with $2.53 \AA$ experimetal data.

Fort he three atoms clusters has been realice divers study for top put in evidence the Jahn-Teller efecto and to calculation the electronics affinity [41-43]. Employed CC has been reported the date for silver clusters with 4, 5 and 6 atoms [44].

\section{Distance in Clusters}

\subsection{Neutral Dimers, Anions and Cations}

How are to observe in the Fig. 1 (Ia), the distancefor neutral cluster of two atoms obtained with HF/LANL1MB is the $2.91 \AA$. Employed the HF/LANL2MB method the distance are to contract $0.1 \AA$, see Fig. 2 (Ia). With HF/LANL2DZ method has been obtained $2.73 \AA$ for the neutral cluster with two atoms, see Fig. 3 (Ia), less who in the two events up mentioned. The difference of $0.18 \AA$ between the cluster optimized with LANL2DZ and LANL1MB, who introduce the relativistic effects are possible for the 19 electrons in the valence layer employed LANLEDZ base, compared with 11 electrons introducted with LANL1MB base and the primitive employed $[45,46]$.

Notwithstanding this values are distant of the experimental data 2.5303(2) Å, see 39].

The dimer structure optimized is to present in the Figure of 1 at 9. The distance is in $\AA$.

$$
\begin{gathered}
\text { a)Ia } \left.{ }^{2.91} \text { b)Ib } 0^{3.27} \mathrm{c}\right) \mathrm{Ic} \\
\mathrm{D}_{\infty h},{ }^{1} \sum_{g}^{+},(0.00,0.45) \mathrm{D}_{\infty h},{ }^{2} \sum_{u}^{+},(0.00,0.57) \mathrm{D}_{\infty h},{ }^{2} \sum_{g}^{+},(0.00,0.42)
\end{gathered}
$$

Fig. 1. The structure of dimer clusters with HF/LANL1MB: a) neutral; b) anions; c) cations. Between parenthesis are reported the relative energy, $\triangle E$, (eV), and biding energy for atom $(\mathrm{eV})$

$$
\begin{gathered}
\text { a)Ia } \left.a^{2.81} \mathrm{o}^{\mathrm{b}) \mathrm{Ib}} \mathrm{a}^{3.08} \mathrm{c}\right) \mathrm{Ic} \mathrm{D}^{3.25} \\
\mathrm{D}_{\infty h}, \sum_{g}^{+},(0.00,0.09) \mathrm{D}_{\infty h},{ }^{2} \sum_{u}^{+},(0.00,0.65) \mathrm{D}_{\infty h},{ }^{2} \sum_{g}^{+},(0.00,0.45)
\end{gathered}
$$

Fig. 2. The structure of dimer clusters with HF/LANL2MB: a) neutral; b) anions; c) cations. Between parenthesis are reported the relative energy, $\triangle E$, (eV), and biding energy for atom $(\mathrm{eV})$

$$
\begin{gathered}
\text { a)Ia } \left.0^{2.73} 0^{\mathrm{b}) \mathrm{Ib}} \mathrm{a}^{2.99} \mathrm{c}\right) \mathrm{Ic} 0^{3.016} \\
\mathrm{D}_{\infty h}, \sum_{g}^{+},(0.00,0.18) \mathrm{D}_{\infty h}, \sum_{u}^{+},(0.00,0.56) \mathrm{D}_{\infty h}, \sum_{g}^{+},(0.00,0.59)
\end{gathered}
$$

Fig. 3. The structure of dimer clusters with HF/LANL2DZ: a) neutral; b) anions; c) cations. Between parenthesis are reported the relative energy, $\triangle E$, (eV), and biding energy for atom (eV)

$$
\begin{gathered}
\text { a)Ia } \left.\left.a^{2.87} \mathrm{~b}\right) \mathrm{Ib} a^{3.19} \mathrm{c}\right) \mathrm{Ic} 0^{3.32} \\
\mathrm{D}_{\infty h}, \sum_{g}^{+},(0.00,0.45) \mathrm{D}_{\infty h},{ }^{2} \sum_{u}^{+},(0.00,0.62) \mathrm{D}_{\propto h}, \sum_{g}^{+},(0.00,0.53)
\end{gathered}
$$

Fig. 4. The structure of dimer clusters with B3LYP/LANL1MB: a) neutral; b) anions; c) cations. Between parenthesis are reported the relative energy, $\triangle E$, (eV), and biding energy for atom $(\mathrm{eV})$

$$
\begin{gathered}
\text { a)Ia } \left.\left.a^{2.74} \mathrm{~b}\right) \mathrm{Ib} a^{2.93} \mathrm{c}\right) \mathrm{Ic} 0^{3.09} \\
\mathrm{D}_{\infty h}, \sum_{g}^{+},(0.00,0.60) \mathrm{D}_{\infty h},{ }^{2} \sum_{u}^{+},(0.00,0.74) \mathrm{D}_{\infty h},{ }^{2} \sum_{g}^{+},(0.00,0.57)
\end{gathered}
$$

Fig. 5. The structure of dimer clusters with B3LYP/LANL2MB: a) neutral; b) anions; c) cations. Between parenthesis are reported the relative energy, $\triangle E$, (eV), and biding energy for atom $(\mathrm{eV})$ 




Fig. 6. The structure of dimer clusters with B3LYP/LANL2DZ: a) neutral; b) anions; c) cations. Between parenthesis are reported the relative energy, $\triangle E$, (eV), and biding energy for atom $(\mathrm{eV})$

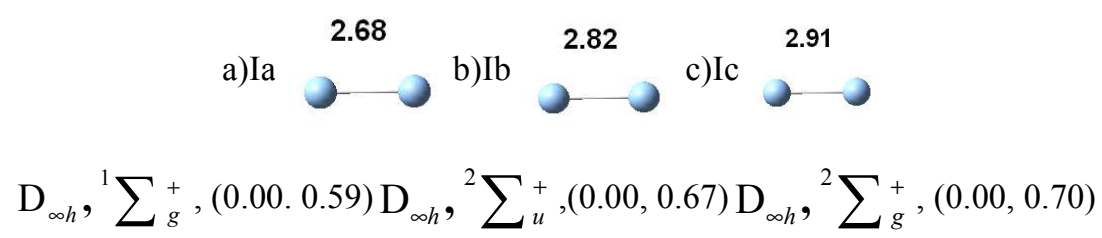

Fig. 7. The structure of dimer clusters with MP2/LANL2DZ: a) neutral; b) anions; c) cations. Between parenthesis are reported the relative energy, $4 E$, (eV), and biding energy for atom $(\mathrm{eV})$

$$
\begin{gathered}
\text { a)Ia } 0^{2.57} \text { b)Ib }{ }^{2.71} \text { c)Ic } \\
\mathrm{D}_{\infty h},{ }^{1} \sum_{g}^{+},(0.70,0.88) \mathrm{D}_{\infty h},{ }^{2} \sum_{u}^{+},(0.00,0.74) \mathrm{D}_{\infty h}, \sum_{g}^{+},(0.00,0.90)
\end{gathered}
$$

Fig. 8. The structure of dimer clusters with PBE/SDD: a) neutral; b) anions; c) cations. Between parenthesis are reported the relative energy, $\triangle E$, (eV), and biding energy for atom $(\mathrm{eV})$

$$
\begin{gathered}
\text { a)Ia }{ }^{2.53} \text { b)Ib }{ }^{2.60} \text { c)Ic }{ }^{2.67} \\
\mathrm{D}_{\infty h}, \sum_{g}^{+},(0.00,1.14) \mathrm{D}_{\infty h}, \sum_{\mathrm{u}}^{+},(0.00,1.07) \mathrm{D}_{\infty h}, \sum_{g}^{+},(0.00,1.08)
\end{gathered}
$$

Fig. 9. The structure of dimer clusters with PBE/3-21G**: a) neutral; b) anions; c) cations. Between parenthesis are reported the relative energy, $\triangle E$, (eV), and biding energy for atom (eV)

For the dimer anion and cation calculated with HF/LANL1MB has been obtained $3.27 \AA$ y $3.37 \AA$, see Fig. 1 (Ib y Ic); the cation cluster to designa an greater distance beteen of the atoms who anion, for the repulsion beteen the nucleos.

The distance beteen the atoms for anion cluster is greater who neutral cluster for the core electrons. Of this manner the electrons of the valence mantle make larger electronic density. Employed DFT with B3LYP the distance beteen the atoms in neutral cluster lower in the following order LANL1MB > LANL2MB > LANL2DZ, see Fig.4 (Ia), Fig. 5 (Ia) y Fig. 6 (Ia). The $2.61 \AA$ obtained with the DFT/B3LYP/LANL2DZ leve is equal who reported in [47] but greater who employed B3P86/LANL2DZ (2.576 $\AA)$ and B3PW91/LANL2DZ $(2.589 \AA$ A $)$ [48]. With SVWN (LSDA)/LANL2DZ and SVWN5/LANL2DZ has been obtained 2.501 y $2.508 \AA$ [48], underestimate with experimental data 2.5303(2) $\AA$ [49]. Fort he neutral cluster of two atoms to employed MP2/LANLEDZ, are obtained $2.68 \AA$ identicaly with [39] and with difference of $0.01 \AA$ obtained with CCSD [39].

For the anion and cation dimer, with the same level, has been obtained $2.91 \AA$ y $2.82 \AA$, similar in the [39].

The value obtained for $\mathrm{Ag}_{2}$ employed PBE/SDD is greater who $0.04 \AA$ of the experimental value and minor with $0.04 \AA$ who obtained with B3LYP/LANL2DZ and $0.01 \AA$ that the value obtained with G96LYP/SDD [39]. In barter, with DFT/PBE/3-21G** level are obtained $2.531 \AA$ similar with the experimental data [49].

Fort he two atoms for anion with the DFT/PBE/3-21G** level has been attained $2.60 \AA$ verynear of the experimental data $2.62 \AA[50]$.

For geometrically to describe the silver dimer cluster I employed PBE and $3-21 \mathrm{G}^{* *}$.

\subsection{Neutral Trimer, Anions and Cations}

For the trimer are not experimental data above the distance of the atoms in clusters, for this reason I present the smallests and sadle point in the potential energy surface for neutral clusters, with B3LYP and LANL1MB, LANL2MB y LANL2DZ bases, with MP2, and DFT/PBE/SDD y DFT/PBE/3-21G**.

Experimentally, [37, 41, 42, 51-55], and employed the thoeretic jobs [32, 44, 56, 57] has been see the Jahn-Teller efects, more for the $D_{3 h}$ structure and are separate in smart, optuse triangle and to draw lines structure [25]. 
In ${ }^{2} E^{\prime}$ the electronic configuration is $\left(a_{1}^{\prime}\right)^{2}\left(e^{\prime}\right)^{1}$ where $a_{1}^{\prime}$ are the combination of three $s \mathrm{AOs}$ and $e^{\prime}$ is the orbital of the not connection double binding [57].

The ${ }^{2} B_{2}$ is always more low in energie that ${ }^{2} A_{1}$, already who the apex atoms mix the caracter of the p orbital and the bonding augmented [58]. The autors of the [19] and [40], employed ab initio han been obtained who $\left(\sigma_{g}\right)^{2}\left(\sigma_{u}\right)^{1}$ is less estable with $0.05 \mathrm{eV}$ who ${ }^{2} E^{\prime}$. The molecular orbitals $\sigma_{g}$, $\sigma_{u} 2 \sigma_{g}$ and as combination of 3 orbitals s of $\mathrm{Ag}_{3}$ are the biding, not biding and antibiding, respectively [17].

Fort he structure trimer neutral the bonding has been obtained with MP2/LANL2DZ, see Fig. 16, and are equal with reported in [39].

The optimized structure is pictored in the following figure, when the biding is in $\AA$.



Fig. 10. The structure of trimer clusters with HF/LANL1MB: a) neutral; b) anions; c) cations. Between parenthesis are reported the relative energy, $\triangle E$, (eV), and biding energy for atom $(\mathrm{eV})$

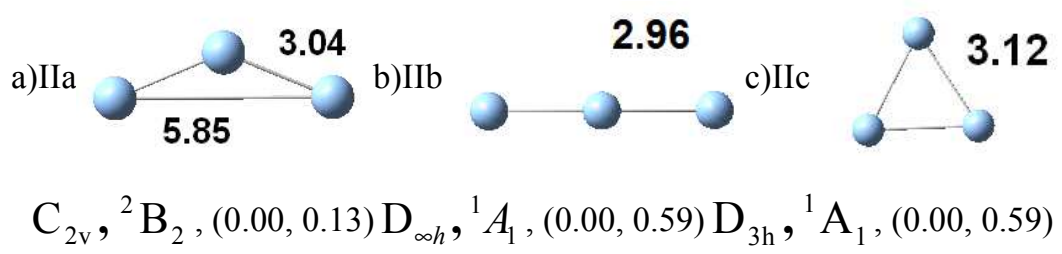

Fig. 11. The structure of trimer clusters with HF/LANL2MB: a) neutral; b) anions; c) cations. Between parenthesis are reported the relative energy, $\triangle E$, (eV), and biding energy for atom $(\mathrm{eV})$

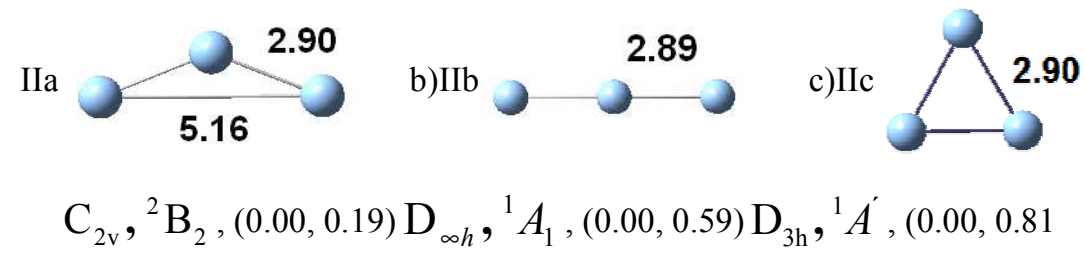

Fig. 12. The structure of trimer clusters with HF/LANL2DZ: a) neutral; b) anions; c) cations. Between parenthesis are reported the relative energy, $\triangle E$, (eV), and biding energy for atom $(\mathrm{eV})$

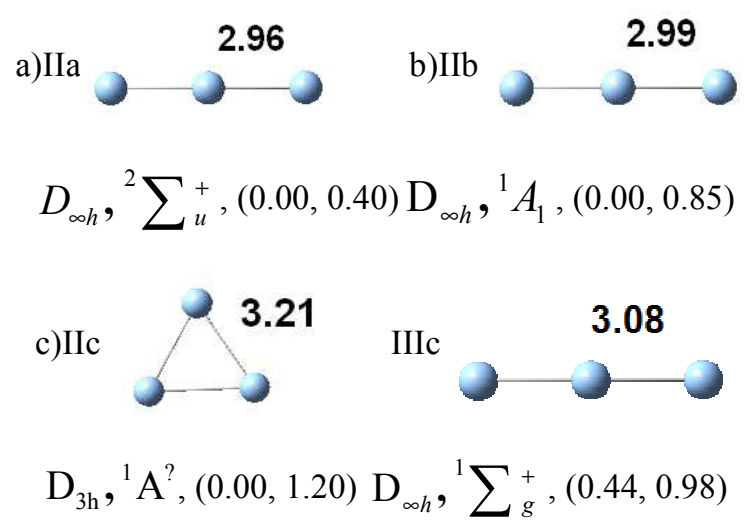

Fig. 13. The structure of trimer clusters with B3LYP/LANL1MB: a) neutral; b) anions; c) cations. Between parenthesis are reported the relative energy, $\triangle E$, (eV), and biding energy for atom (eV)

a)IIa

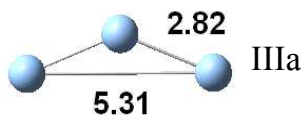




$$
\begin{aligned}
& \mathrm{C}_{2 \mathrm{v}},{ }^{2} A^{\prime},(0.00,0.55) \mathrm{D}_{\infty h},{ }^{1} A_{1},(0.01,0.55) \\
& \mathrm{D}_{\infty h},{ }^{1} A_{1},(0.00,1.05) \mathrm{D}_{3 \mathrm{~h}},{ }^{1} \mathrm{~A}^{?},(0.00,1.51)
\end{aligned}
$$

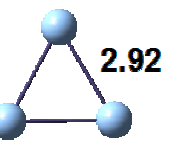

Fig. 14. The structure of trimer clusters with B3LYP/LANL2MB: a) neutral; b) anions; c) cations. Between parenthesis are reported the relative energy, $\triangle E$, $(\mathrm{eV})$, and biding energy for atom $(\mathrm{eV})$

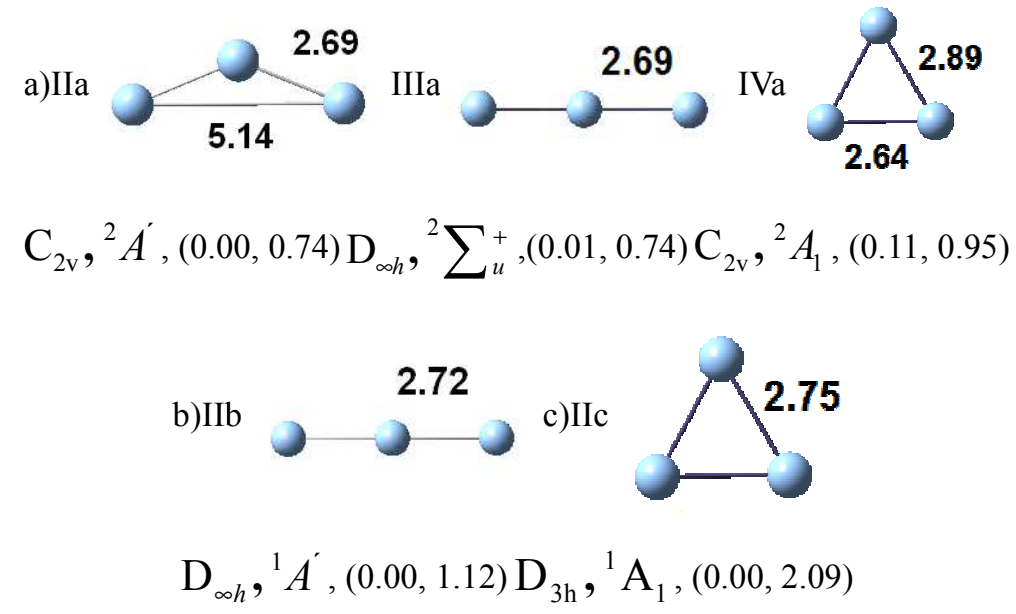

Fig. 15. The structure of trimer clusters with B3LYP/LANL2DZ: a) neutral; b) anions; c) cations. Between parenthesis are reported the relative energy, $\triangle E$, $(\mathrm{eV})$, and biding energy for atom $(\mathrm{eV})$

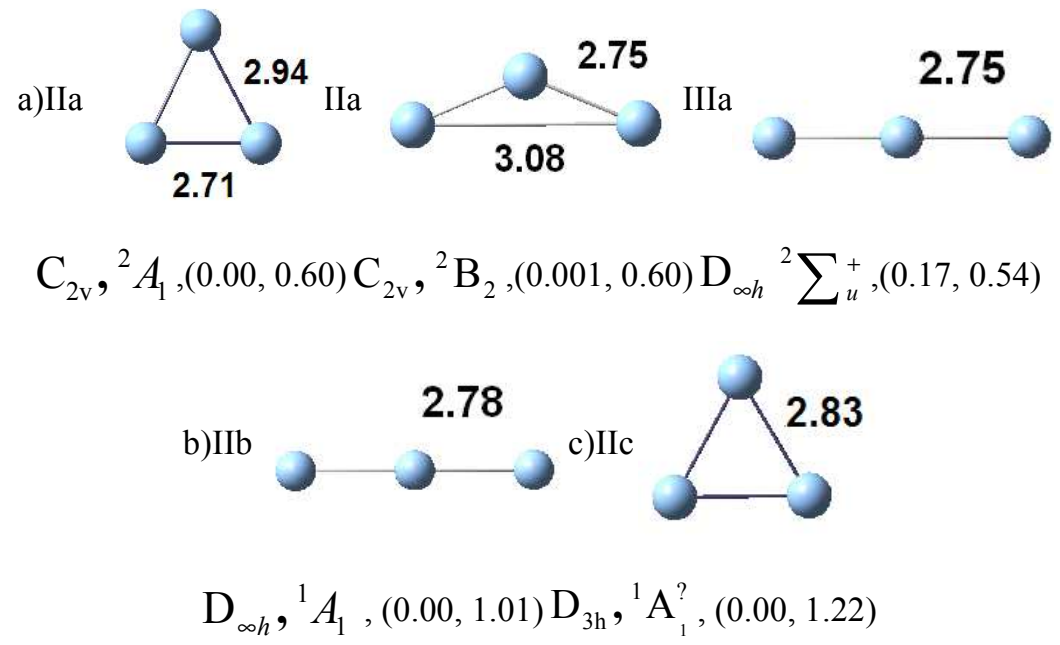

Fig. 16. The structure of trimer clusters with MP2/LANL2DZ: a) neutral; b) anions; c) cations. Between parenthesis are reported the relative energy, $\triangle E$, (eV), and biding energy for atom $(\mathrm{eV})$

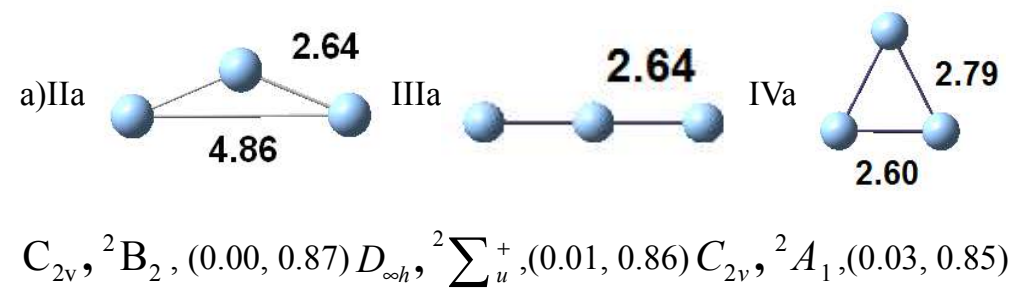




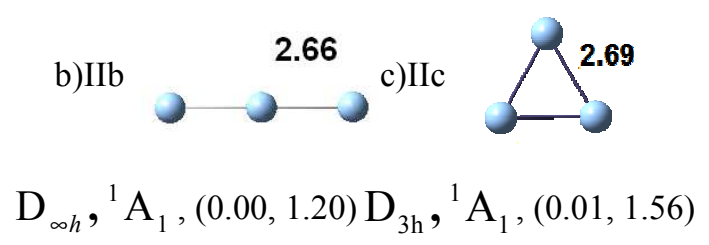

Fig. 17. The structure of trimer clusters with PBE/SDD: a) neutral; b) anions; c) cations. Between parenthesis are reported the relative energy, $\triangle E$, (eV), and biding energy for atom $(\mathrm{eV})$

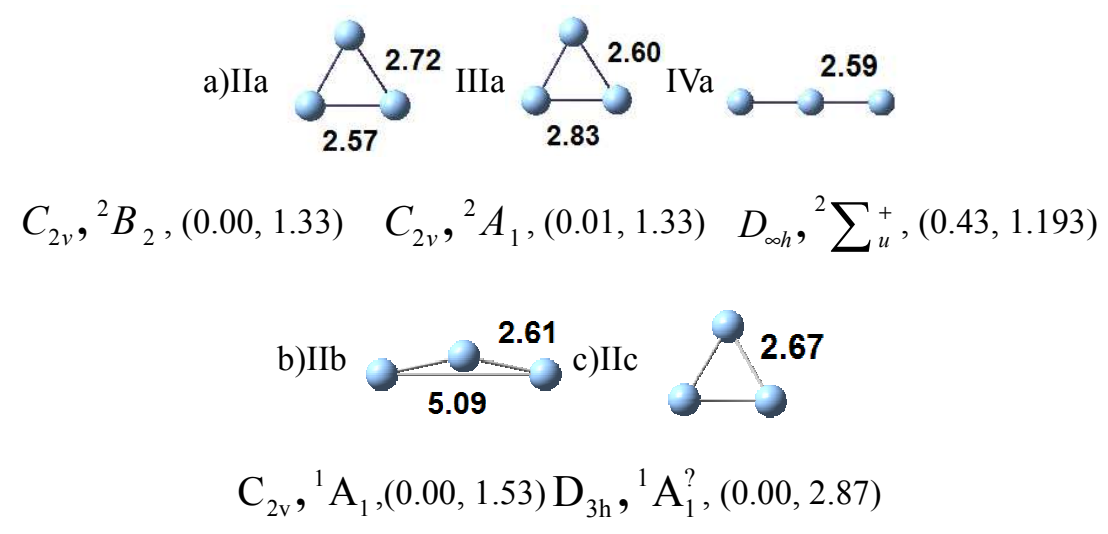

Fig. 18. The structure of trimer clusters with PBE/3-21G**: a) neutral; b) anions; c) cations. Between parenthesis are reported the relative energy, $\triangle E$, (eV), and biding energy for atom $(\mathrm{eV})$

The structure of obtuse triangle otimized with HF/LANL1MB are the angle $148.74^{\circ}$ and the distances 3.21 and $6.18 \AA$, see Fig. 10 (IIa).

Used the HF/LANL2DZ for the 3 atoms clusters exist also the contraction of the bonding for the relativistic effect, see Fig. 3.13 (IIa).

Analize the Fig. 17 (IIa) y 18 (IIa) I observed who the electronic correlation effects are competitivlly with the relativist effects for trimer structure has been the difference for the biding of 0.04 and $2.03 \AA$. Moreover, compared with B3LYP/LANL2DZ where are obtained 2.69 SDD and PBE to influence of geometrical property of $\mathrm{Ag}_{3}$.

Except when are employed PBE/3-21G** the anion cluster are for all levels of theory the linear geometry.

For the anion cluster with DFT/PBE/3-21G** has been obtained the triangular structure lees stable by $1.3 \mathrm{eV}$ who linear structure [51].

To compare the DFT/B3LYP and HF, and employed LANL2DZ, have been obtained the difference of $0.21 \AA$, see Fig. 15 (IIIa) y Fig. 12 (IIIa).

In the case of $\mathrm{Ag}_{3}$ the electronic correlation efects alone or complementary with relativistic effects (PBE/SDD) are strived. For anions structures the more stable are the lineal structure, for neutral structure the obtuse-angled triangle and for the cation the equilateral triangle.

\subsection{Tetramer and Pentamer Neutral, Anions and Cations}

The $\mathrm{Ag}_{4}$ structure are HOMO doubly ocuped in the singlete.. Compared the rhombic structure of neutral cluster in la Fig. 21 (IIIa) and the catión cluster, see Fig. 21 (IVc), optimized with HF/LANL2DZ, I observ who the distance are minor for neutral cluster who cation cluster $(2.77$ y $2.83 \AA)$.

Beteen two central atoms, possibly on account of in part of the $\mathrm{p}$ atomic orbitals to bind weakly in HOMO with another atoms allowances above the greater diagonal. For such motive in the neutral cluster doubly occupied in HOMO the distance beteen two atoms are less who in the cation.

The same to present for the silver cultir when are performed with PBE/SDD, see Fig. 26 (IIIa y IIIc) and the optimized cluster with MP2, see Fig. 25 (IIIa y IIIc).

Fort he romboidal structure, the experimental distance are reported in [39] is $2.79 \AA$ comparison with $2.81 \AA$ obtained in the present work, see Fig. 24 (IIIa). The form $\mathrm{T}$ is very stable, successively for romboidal and lineal structure.
a)IIIa



$3.28 \quad 2.87$ 


$$
\mathrm{C}_{2 \mathrm{v}},{ }^{1} \mathrm{~A}_{1},(0.00,0.02) \mathrm{D}_{\infty h},{ }^{1} \sum_{g}^{+},(0.049,0.006)
$$

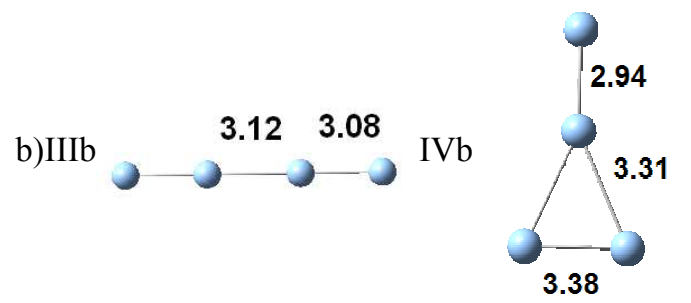

$$
\mathrm{D}_{\text {och }}{ }^{2} \mathrm{~A},(0.196,0.05) \mathrm{C}_{2 \mathrm{v}},{ }^{2} \mathrm{~A}^{?},(0.00,0.54)
$$

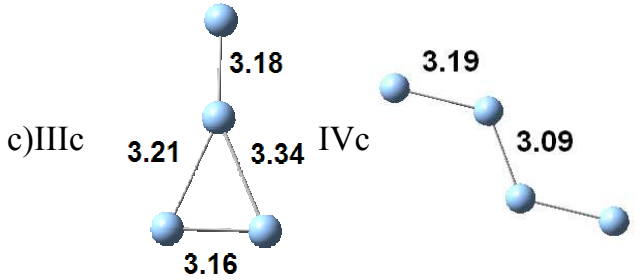

$$
\mathrm{C}_{\mathrm{s}},{ }^{2} \mathrm{~A}^{?},(0.00,0.45) \mathrm{C}_{2 \mathrm{~h}},{ }^{2} \mathrm{~A}^{?},(0.001,0.45)
$$

Fig. 19. The structure of $A g_{4}$ clusters with HF/LANLIMB: a) neutral; b) anions; c) cations. Between parenthesis are reported the relative energy, $\triangle E$, (eV), and biding energy for atom (eV)



$$
\mathrm{C}_{2 \mathrm{v}},{ }^{1} \mathrm{~A}^{?},(0.00,0.13) \mathrm{D}_{\infty h}, \sum_{g}^{+},(0.00,0.62)
$$



$$
\mathrm{C}_{2 \mathrm{v}},{ }^{2} \mathrm{~A}^{?}(0.18,0.76) \mathrm{C}_{2 \mathrm{v}},{ }^{2} \mathrm{~A}^{?},(0.00,0.54)
$$

Fig. 20. The structure of $\mathrm{Ag}_{4}$ clusters with HF/LANL2MB: a) neutral; b) anions; c) cations. Between parenthesis are reported the relative energy, $\triangle E$, (eV), and biding energy for atom $(\mathrm{eV})$

a)IIIa

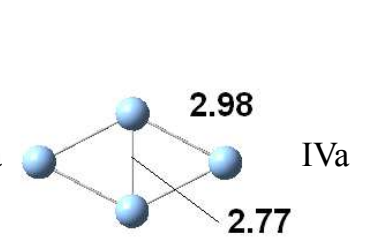

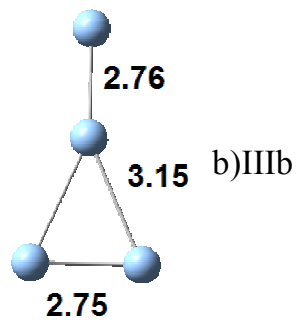

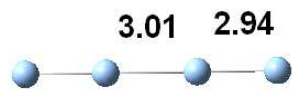




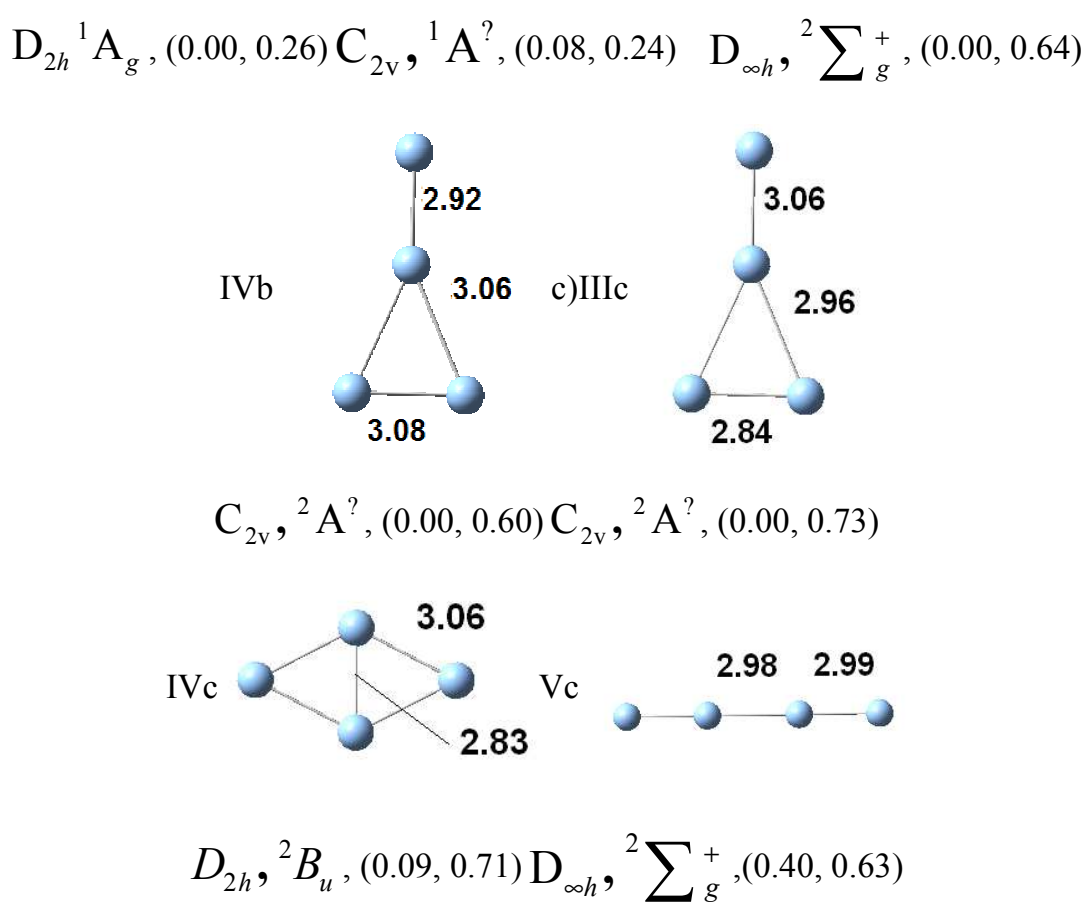

Fig. 21. The structure of $\mathrm{Ag}_{4}$ clusters with HF/LANL2DZ: a) neutral; b) anions; c) cations. Between parenthesis are reported the relative energy, $\triangle E$, (eV), and biding energy for atom (eV)



Fig. 22. The structure of $\mathrm{Ag}_{4}$ clusters with B3LYP/LANLIMB: a) neutral; b) anions; c) cations. Between parenthesis are reported the relative energy, $\Delta E$, (eV), and biding energy for atom $(\mathrm{eV})$






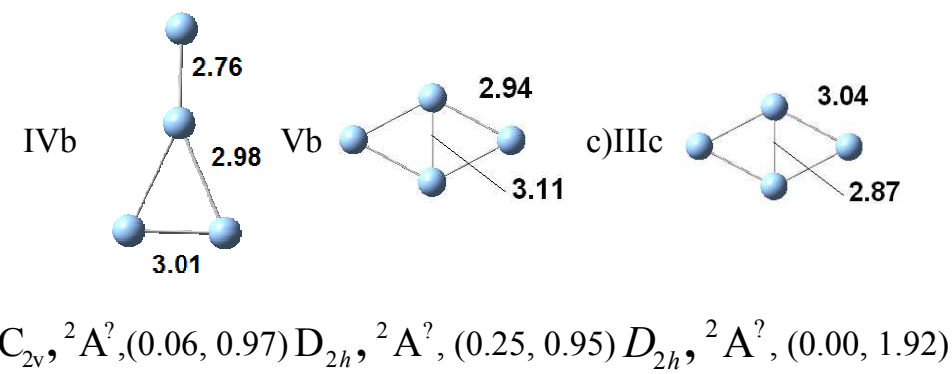

Fig. 23. The structure of $A_{4}$ clusters with B3LYP/LANL2MB: a) neutral; b) anions; c) cations. Between parenthesis are reported the relative energy, $\triangle E$, (eV), and biding energy for atom $(\mathrm{eV})$

a)IIIa

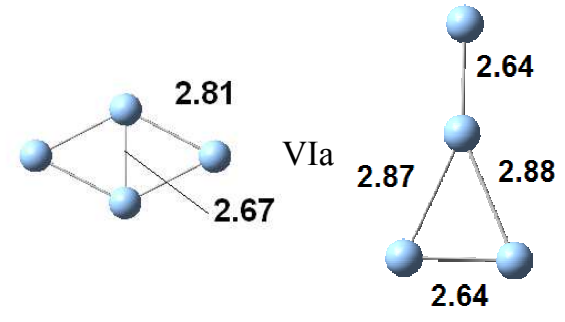

$\mathrm{D}_{2 h},{ }^{1} \mathrm{~A}^{?},(0.00,0.95) \mathrm{C}_{2 \mathrm{~s}},{ }^{1} \mathrm{~A}^{?},(0.11,0.93)$

b)IIIb


$\mathrm{D}_{\infty h},{ }^{2} \sum_{g}^{+},(0.00,1.11) \mathrm{C}_{2 \mathrm{v}},{ }^{2} \mathrm{~A}^{?},(0.09,1.09)$

c)IIIc

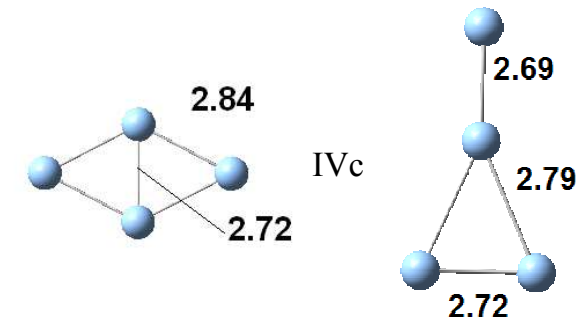

$$
D_{2 h},{ }^{2} \mathrm{~A}^{\prime},(0.00,2.57) \mathrm{C}_{2 \mathrm{v}},{ }^{2} \mathrm{~A}^{?},(0.13,2.51)
$$

Fig. 24. The structure of $\mathrm{Ag}_{4}$ clusters with B3LYP/LANL2DZ: a) neutral; b) anions; c) cations. Between parenthesis are reported the relative energy, $\triangle E$, (eV), and biding energy for atom $(\mathrm{eV})$








Fig. 25. The structure of $\mathrm{Ag}_{4}$ clusters with MP2/LANL2DZ: a) neutral; b) anions; c) cations. Between parenthesis are reported the relative energy, $\triangle E$, (eV), and biding energy for atom $(\mathrm{eV})$

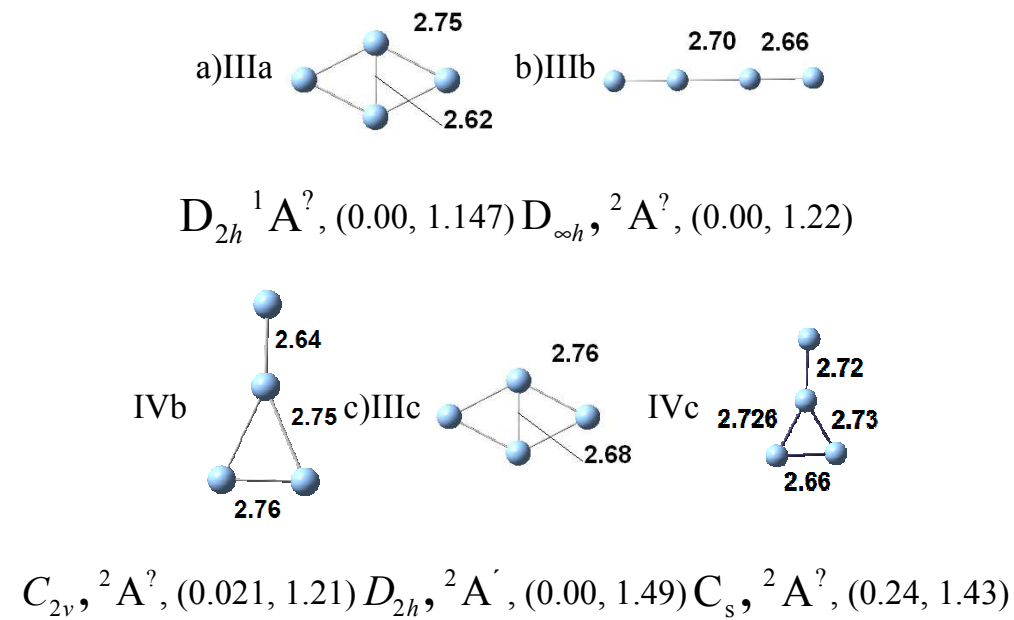

Fig. 26. The structure of $\mathrm{Ag}_{4}$ clusters with PBE/SDD: a) neutral; b) anions; c) cations. Between parenthesis are reported the relative energy, $\triangle E$, (eV), and biding energy for atom (eV)

a) IIIa

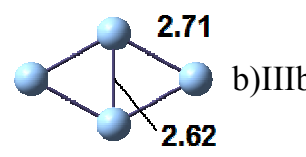

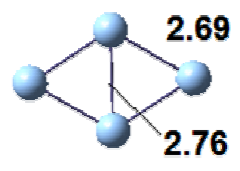

2.76
2.73

c)IIIc

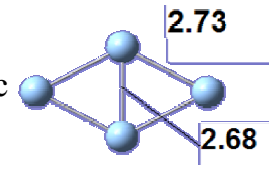

$$
\mathrm{D}_{2 h},{ }^{1} \mathrm{~A}^{?}(0.00,1.69) D_{2 h},{ }^{2} \mathrm{~A}^{\prime}(0.00,1.84) D_{2 h},{ }^{2} \mathrm{~A}^{\prime},(0.00,3.89)
$$

Fig. 27. The structure of $\mathrm{Ag}_{4}$ clusters with PBE/3-21G**: a) neutral; b) anions; c) cations. Between parenthesis are reported the relative energy, $\triangle E$, (eV), and biding energy for atom (eV)

In [44] has been reportade the distance for $\mathrm{Ag}_{4}^{-}$anions cluster obtained withSCF 1e-RECP-CVC. Romboedral the $2.789 \AA$ and $2.838 \AA$ with difference of $0.02 \AA$ of the distance obtained optimized with PBE/SDD, but less who $2.69 \AA$ obtained with DFT and PBE AND 3-21G**.

For $\mathrm{Ag}_{4}$ neutral cluster are performed with HF/LANL2DZ and B3LYP/LANL2DZ
Comparison the distance beteen atoms in the neutral cluster with rombic structure optimized rebound who contraction for electronic correlation is $0.17 \AA$ and $0.10 \AA$, see Fig. 21 y 24 (IIIa y IIIa).

For cations cluster in Fig. 21 and 24 (IIIb, IVb, IIIb y IIIc) the the contraction are $0.25 \AA$, greater who in the neutral clusters.

Beteen the neutral structure optimized with 5 atoms with 
DFT/B3LYP/LANL2DZ, the trpezium es better stable who piramidal with squere base so how are moderate in [18] how

experimental date.
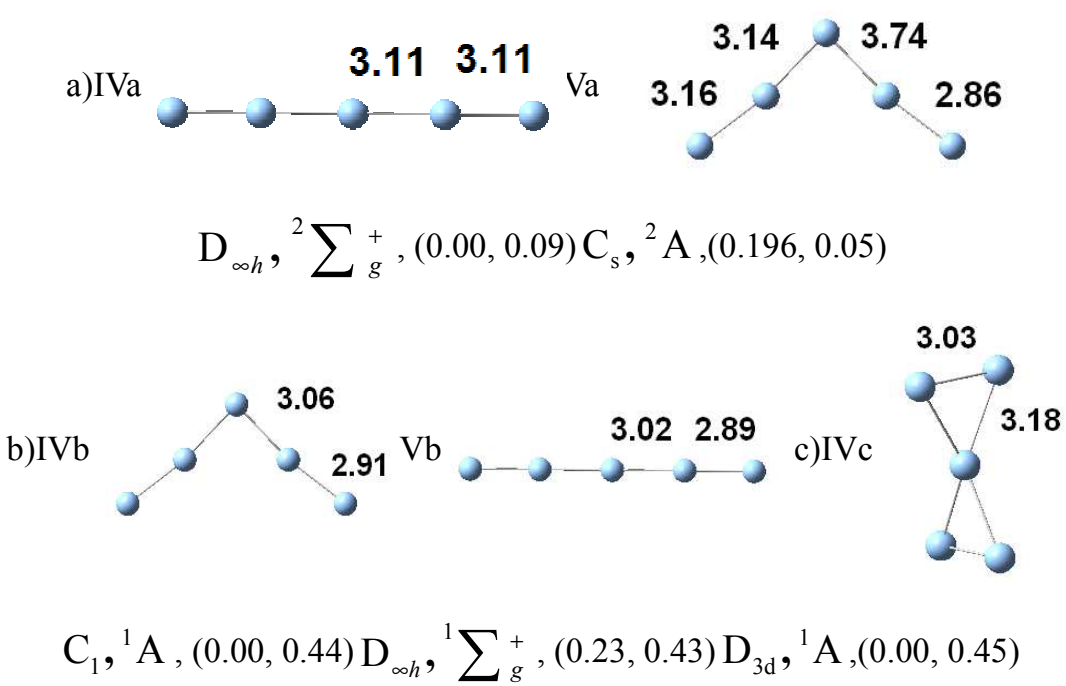

Fig. 28. The structure of $A g_{5}$ clusters with HF/LANL1MB: a) neutral; b) anions; c) cations. Between parenthesis are reported the relative energy, $\triangle E$, (eV), and biding energy for atom $(\mathrm{eV})$

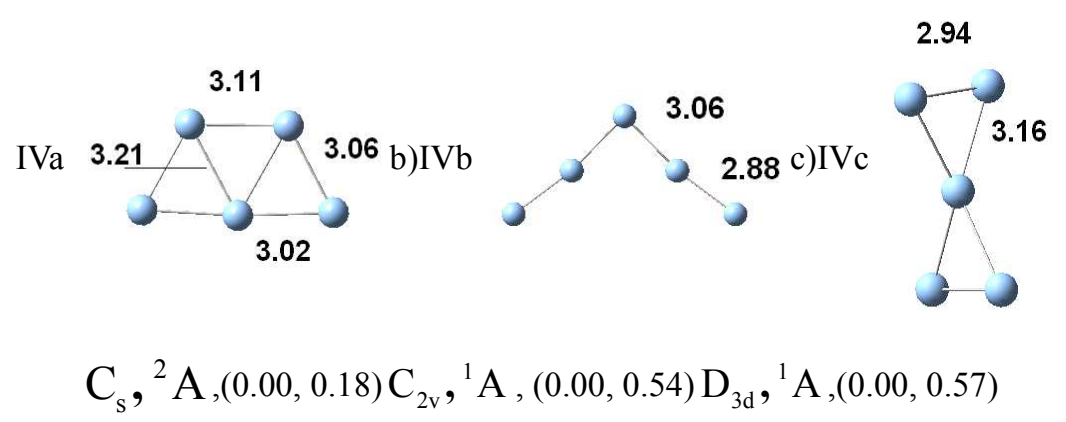

Fig. 29. The structure of $A g_{5}$ clusters with HF/LANL2MB: a) neutral; b) anions; c) cations. Between parenthesis are reported the relative energy, $\Delta E$, (eV), and biding energy for atom (eV)

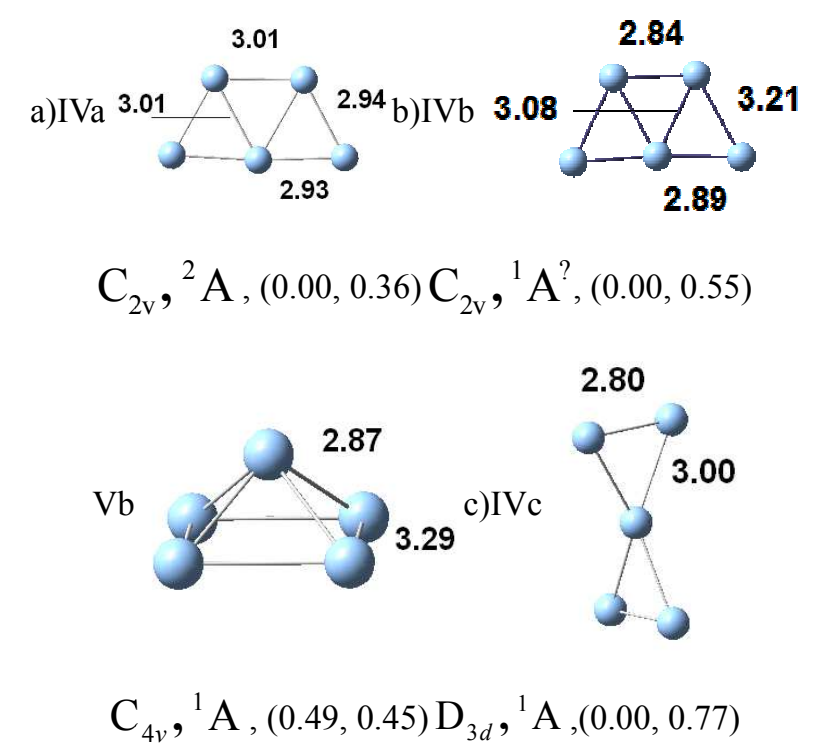

Fig. 30. The structure of $A g_{5}$ clusters with HF/LANL2DZ: a) neutral; b) anions; c) cations. Between parenthesis are reported the relative energy, $\Delta E$, (eV), and biding energy for atom $(\mathrm{eV})$ 
a)IVa



$\mathrm{C}_{\mathrm{s}},{ }^{2} \mathrm{~A}^{?},(0.00,0.47) \mathrm{D}_{\infty h}, \sum_{g}^{+},(0.00,0.87) \mathrm{D}_{3 \mathrm{~d}},{ }^{1} \mathrm{~A},(0.00,2.23)$

Fig. 31. The structure of $A g_{5}$ clusters with B3LYP/LANL1MB: a) neutral; b) anions; c) cations. Between parenthesis are reported the relative energy, $\triangle E$, (eV), and biding energy for atom $(\mathrm{eV})$

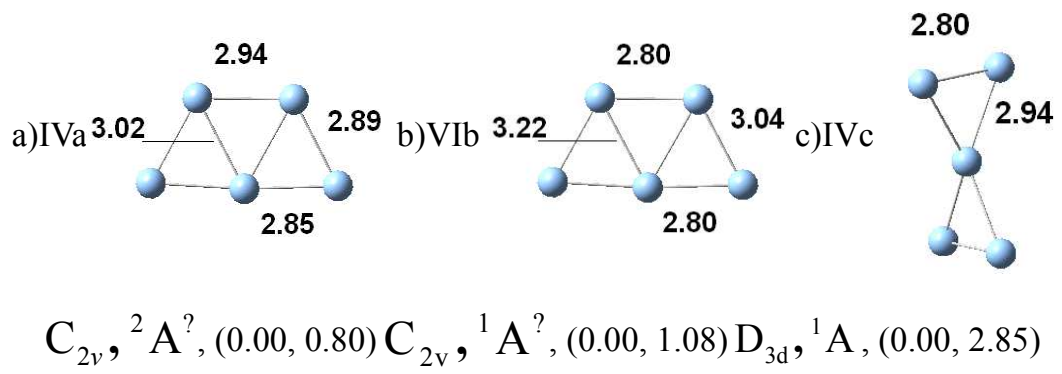

Fig. 32. The structure of $A g_{5}$ clusters with B3LYP/LANL2MB: a) neutral; b) anions; c) cations. Between parenthesis are reported the relative energy, $\triangle E$, (eV), and biding energy for atom $(\mathrm{eV})$

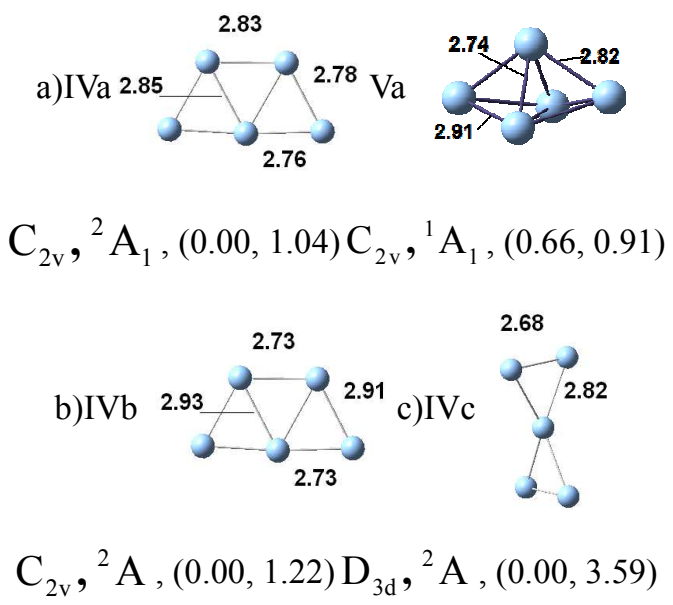

Fig. 33. The structure of $\mathrm{Ag}_{5}$ clusters with B3LYP/LANL2DZ: a) neutral; b) anions; c) cations. Between parenthesis are reported the relative energy, $\triangle E$, (eV), and biding energy for atom $(\mathrm{eV})$

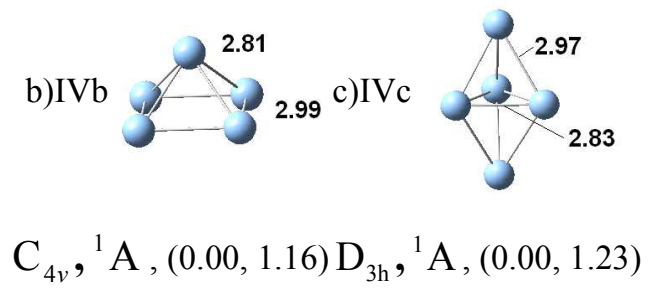

Fig. 34. The structure of $A g_{5}$ clusters with MP2/LANL2DZ: a) neutral; b) anions; c) cations. Between parenthesis are reported the relative energy, $\triangle E$, (eV), and biding energy for atom $(\mathrm{eV})$

I oberve the influyence of the relativistic effects for $\mathrm{Ag}_{5}^{+}$ employed HF/LANL1MB, HF/LANL2MB and HF/LANL2DZ with the contraction of 0.09 and $0.06 \AA$ in distance, see Fig. 38 (IVc), 29 (IVc) and 30 (IVc). With
B3LYP the difference beteen distance in $\mathrm{C}_{2 v}, \mathrm{~A}_{1}$ is the 0.16 $\AA$ with $\mathrm{Ag}_{5}$ optimized with HF/LANL2DZ, see Fig. 21 (Va) and Fig. 33 (IVa).

On change compataively the variation of disstance beteen 
the structure optimized trapezoidal with B3LYP/LANL1MB and B3LYP/LANL2DZ the difference are $0.26 \AA$ who to oppose who relativist effect are greater who the electronic correlation, the plan estructure has been more stable when the bipiramydal structure with triangle base, so report in experimental data [18].

This debit of hybridization beteen $4 d$ y $5 s$ that to favor the planar structure. The hibridization are interesinteresting for the relativistic effects in $\mathrm{Au}, \mathrm{Cu}$ and silver, in this case particular [59].

Fort he silver plata $\mathrm{Ag}_{7}$ rebound to be who the planar structure is stable with diminution in distance, so how $A u_{7}$ [67].

\section{Biding Energy for Atom}

Is important to study the biding energy for atom because to refer at stability of clusters. Are graphical the value of the biding energy of the neutral cluster, anions and cations Fig. 35-40 calculated with $\frac{E_{b}}{n}=\left(n E_{1}-E_{n}\right) / n$ where $E_{1}$ and $E_{n}$ are the total energy for the neutral species optimizated for un atom and $\mathrm{n}$ atoms; $\frac{E^{ \pm}}{n}=\left[(n-1) E_{1}+E_{1}^{ \pm}\right] / n$ where $E_{1}^{ \pm}$and $E_{n}^{ \pm}$are the total energy for the positive and negative for un atom and $\mathrm{n}$ atoms.

In Fig. 35 are presented the reults obtained for the neutral clusters of $\operatorname{Ag}_{n}(n=1-5)$. Tendeney in the values of biding energy of the cluster augment with the number of atoms employed the HF/LANL2DZ level. Th estructure $\mathrm{T}$ for $\mathrm{Ag}_{4}$ optimized with LANL2MB and LANL1MB is less stable who the triangular, rombic and leneal structure

In Jahn=Teller for the neutral clusters $\mathrm{Ag}_{3}$ optimized with HF/LANL2DZ resulte who el isomer more stable are the optuse trinagle with the biding energy with $0.193 \mathrm{eV}$, successive for the leneal structure with biding energy of $0.191 \mathrm{eV}$ and, for the last is the $C_{2 v},{ }^{2} A_{1}$ structure with 0.161 eV, see Fig. 32 (IIa, IIIa y IVa), when this are not the experimental reported where the ${ }^{2} B_{2}$ is better stable who ${ }^{2} A_{1}$ $[48,50]$.

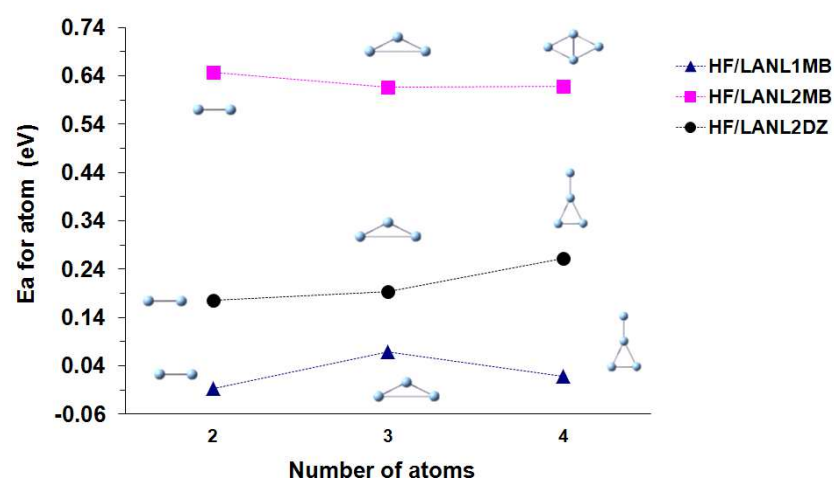

Fig. 35. Biding energy for atom for neutral clusters employed HF. VS. Number of atom for clusters. The mast stable structure.
In the Fig. 19 has been optimized the $D_{\infty h}, \sum_{g}^{+}$lineal with $\mathrm{HF} / \mathrm{LANL} 1 \mathrm{MB}$, for $\mathrm{Ag}_{4}$ and are the value $0.013 \mathrm{~V}$ who the T form with $C_{2 v},{ }^{1} A_{1}$ simmetryFig. 19 (IIIa).

For $\mathrm{Ag}_{4}$ optimized with HF/LANL2DZ the structure more stableis rombic with the little diference $0.12 \mathrm{eV}$, for biding energy, with the T form with $C_{2 v}{ }^{1}{ }^{1} A_{1}$ simmetry, see Fig. 21

For anions clusters, see Fig.36, for $\mathrm{Ag}_{3}$ and $\mathrm{Ag}_{4}$, the form more stable are lineal. For Ag4 anion optimized with LANL1MB the lineal form is more stable who $\mathrm{T}$ form with 0.08 eV only see Fig. 19.

The distribution of charge after to remove 1 electron for $\mathrm{Ag}_{4}^{+}$to favor the $\mathrm{T}$ form how more stable with biding energy $0.73 \mathrm{eV}$, the act of following for rombic and lineal structure with 0.71 and $0.63 \mathrm{eV}$ (see Table 3.9).

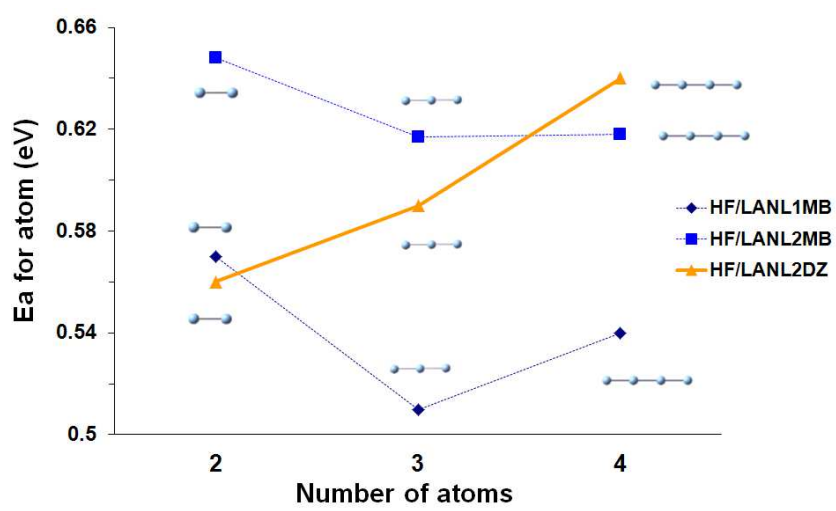

Fig. 36. Biding energy for atom for neutral clusters employed HF. Vs. Number of atom for clusters. The mast stable structure.

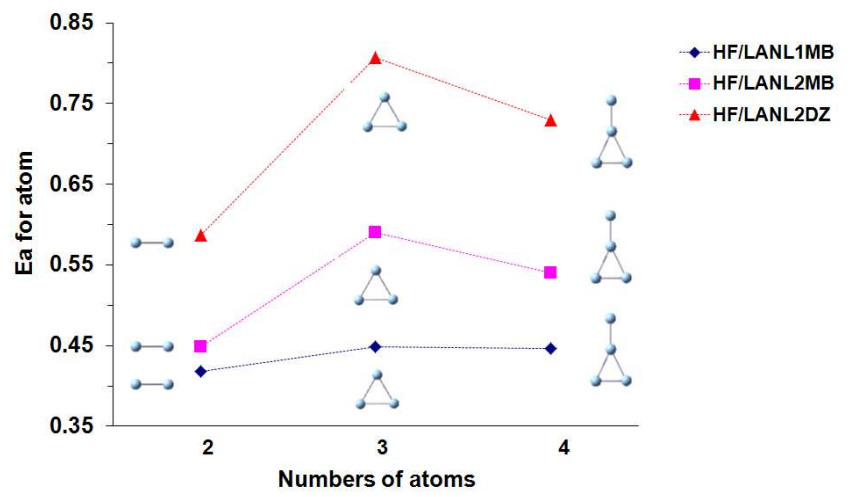

Fig. 37. Biding energy for atom for neutral clusters employed HF. Vs. Number of atom for clusters. The mast stable structure.

In the Fig. 38 are graphicate the value of biding energy for the neutral of silver with 2 and 4 atoms. When are optimized the cluster with B3LYP/LANL1MB han been optained lees value for $\mathrm{Ag}_{\mathrm{n}}(\mathrm{n}=2-4) 0.446 \mathrm{eV}, 0.403 \mathrm{eV}$ and $0.511 \mathrm{eV}$, see Fig. 38

The value of biding energy for dimer clusters obtained with PBE/SDD y B3LYP/LANL2DZ of $0.876 \mathrm{eV}$ and 0.776 eV near of the data experimental [60].

The for biding energy obtained for the isomer ${ }^{2} B_{2},{ }^{2} \sum_{u}^{+}$ and ${ }^{2} A_{1}$ obtained $\mathrm{Ag}_{3}$ with B3LYP/LANL2DZ has been 
$0.740 \mathrm{eV}, 0.705$ y $0.738 \mathrm{eV}$.

In this case has been defered to the experimentals results where the diference beteen ${ }^{2} B_{2}$ and ${ }^{2} A_{1}$ are the $0.022 \mathrm{eV}$ [61] and, beteen ${ }^{2} B_{2}$ y ${ }^{2} \sum_{u}^{+}$are $0.15 \mathrm{eV}$ in [38] or the value 0.85 $\mathrm{eV}$ reported in [48].

When has been optimized with MP2/LANL2DZ the difference beteen ${ }^{2} A_{1}$ and ${ }^{2} B_{2}$ is the $0.001 \mathrm{eV}$ only the biding energy are the same $0.6 \mathrm{eV}$. For PBE/3-21G** has been see greater values, see Fig. 38, and the biding energy concured with the report in the literatura [60].

For $\mathrm{Ag}_{4}^{-}$optimized with B3LYP/LANL2MB the $D_{\infty h},{ }^{2} \sum_{g}^{+}$ are biding energy for atom of $1.01 \mathrm{eV}$ compared with de $\mathrm{T}$ form who are $0.97 \mathrm{eV}$ and $D_{2 h},{ }^{2} B_{2 u}$ with $0.95 \mathrm{eV}$ (see Fig. 23).

Employed PBE/SDD level of theory for optained $\mathrm{Ag}_{4}^{-}$the difference beteen lineal structure end $\mathrm{T}$ form are $0.01 \mathrm{eV}$, implicated who energy for separation the two structure is almost equal (see Table 3.27). On the contrary, employed MP2/LANL2DZ level of theorie in the Fig. 25 see who lineal structure $\mathrm{Ag}_{4}^{-}$art he biding energy minor $(0.96 \mathrm{eV})$ who structure $D_{2 h},{ }^{2} B_{2}$ with $0.99 \mathrm{Ev}$ and rombic structure $D_{2 h},{ }^{2} B_{2 u}$ with $1.13 \mathrm{eV}$.

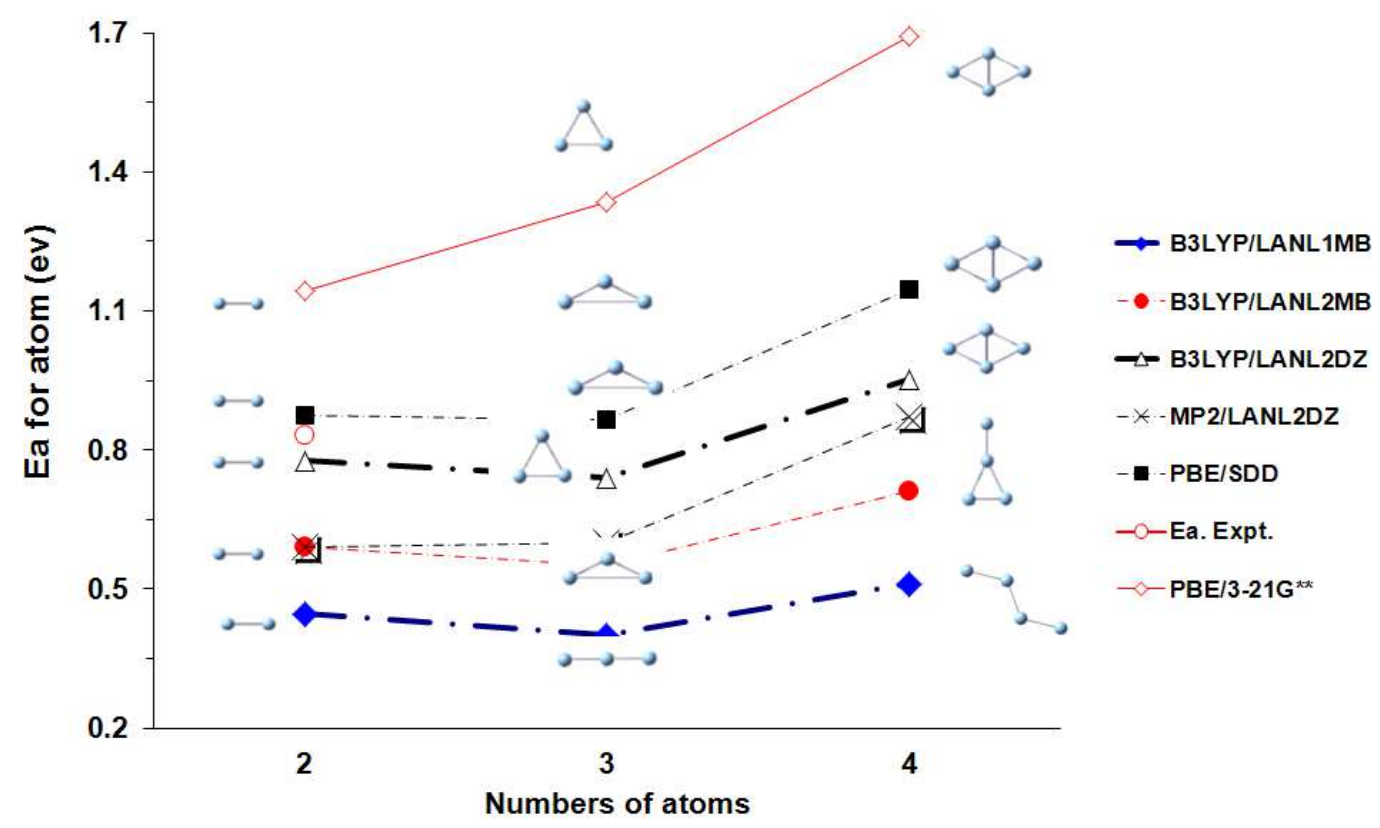

Fig. 38. Biding energy for atom for neutral clusters employed DFT. Vs. Number of atom for clusters. Eaexperimental [36].The mast stable structure.

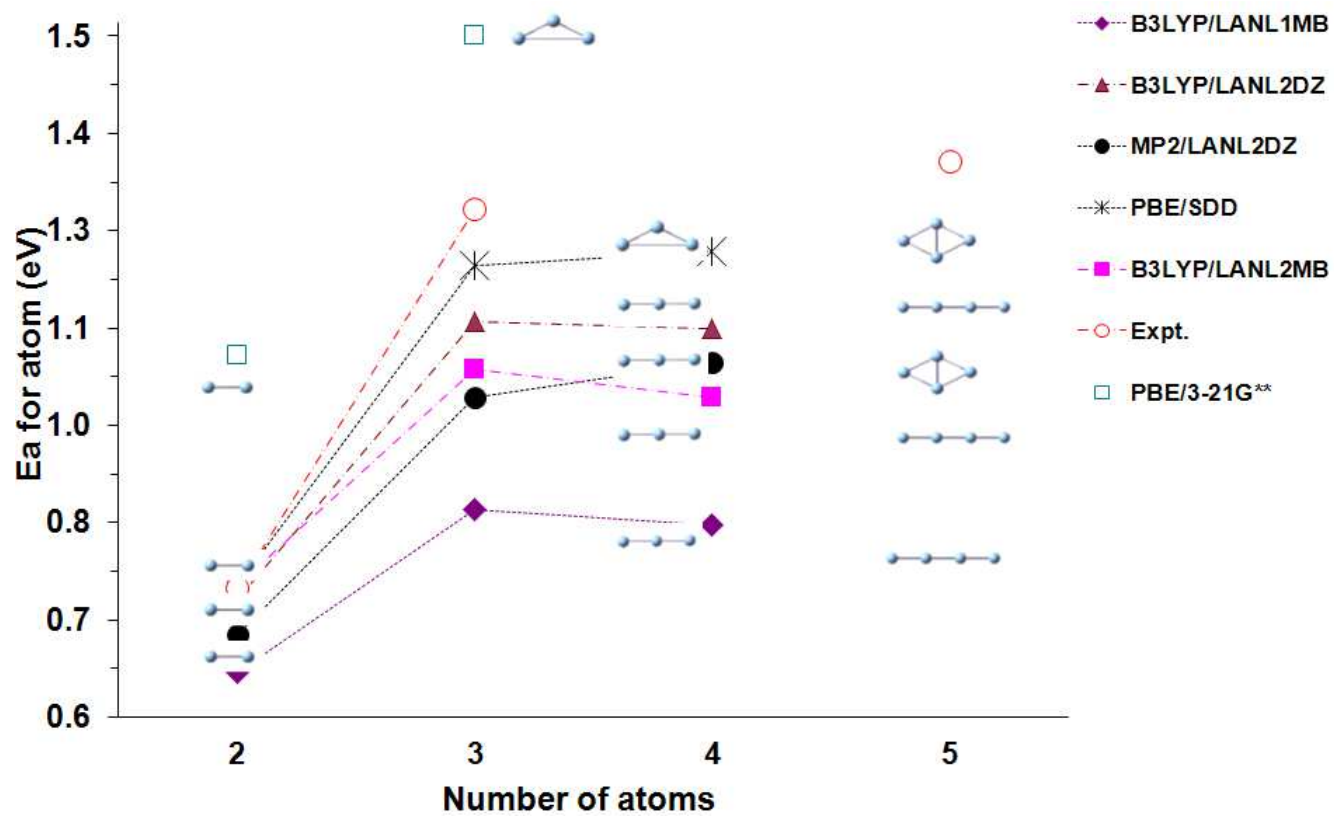

Fig. 39. Biding energy for atom for neutral clusters employed DFT. Vs. Number of atom for clusters. Ea experimental [36].The mast stable structure. 




Fig. 40. Biding energy for atom for neutral clusters employed DFT. Vs. Number of atom for clusters. The mast stable structure.

\section{Ionization Potential and Electronic Affinity}

\subsection{Adiabatic Electronic Affinity and Vertical}

Inthis section are show the values obtained for electronic affinitie and ionization potential for compared with the theoric result and experimental date and see which of the effects relativistic or electronic correlation interfer in this proprieties.

The calculus for the electronic affinities has been calculated with $A=E_{n}-E_{n}^{-}$, where $E_{n}$ is the total energy for the neutral species optimized and $E_{n}^{-}$is the total energy for the anion species after optimized.
How to observe in the Fig. 41 and 42 the values for electronic affinity are distant for the experimental data and are not the same tendency for the clusters optimized with HF and LANL1MB, LANL2MB and LANL2DZ bases.

This a shop sign who HF are not describet fitly the adiabatic electronic affinity for the silver clusters alredy who solely consider the relativistic effects and giving who understimade the difference of energy of the $A g_{n}$ and $\mathrm{Ag}_{n}^{-}$.

The difference for the silver clusters $\mathrm{Ag}_{3}$ are $1.5 \mathrm{eV}$ for $\mathrm{Ag}_{2}$ are $0.43 \mathrm{eV}$ and $\mathrm{Ag}_{4} 0.32 \mathrm{eV}$, see Fig. 45 .

Compared Fig. 41 and 43 has been observed who the results obtained for vertical electronic affinity concured with the adiabatic electronic affinitie motive for which is advisable employed vertical electronic affinity.



Fig. 41. Adiabatic electronic afinity for silver cluster employed. Vs. Number of atoms in the cluster. AEA1 experimental [61-63]. AEA2 experimental [37]. 


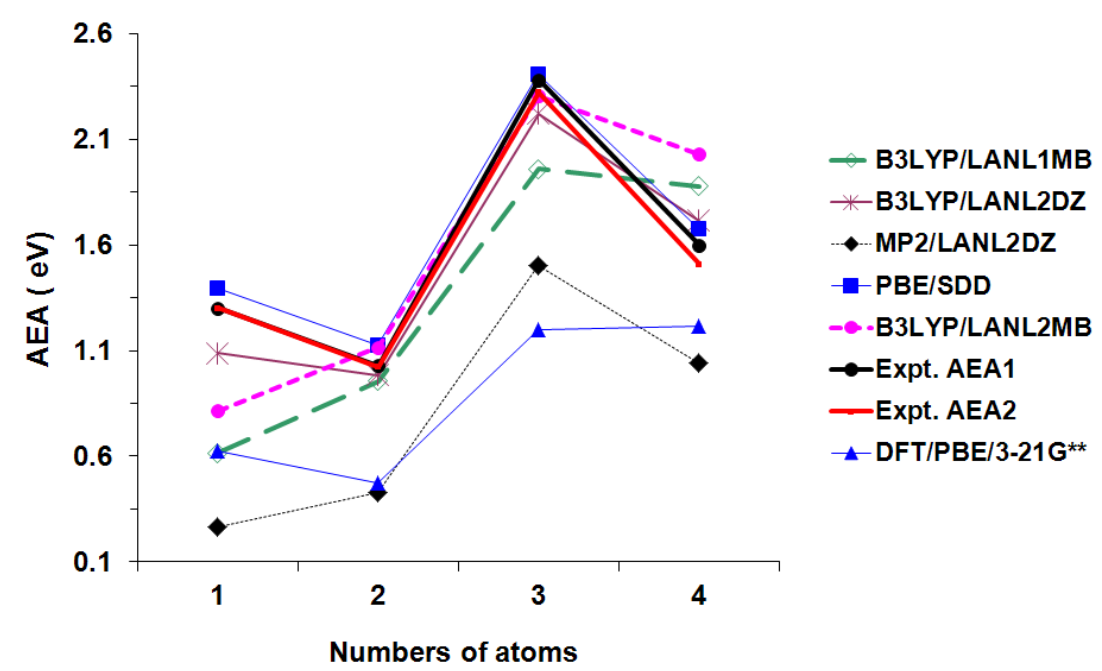

Fig. 42 Adiabatic electronic afinity for silver cluster employed DFT. Vs. Number of atoms in the cluster.. AEA1 experimental [61, 63]. AEA2 experimental[37]

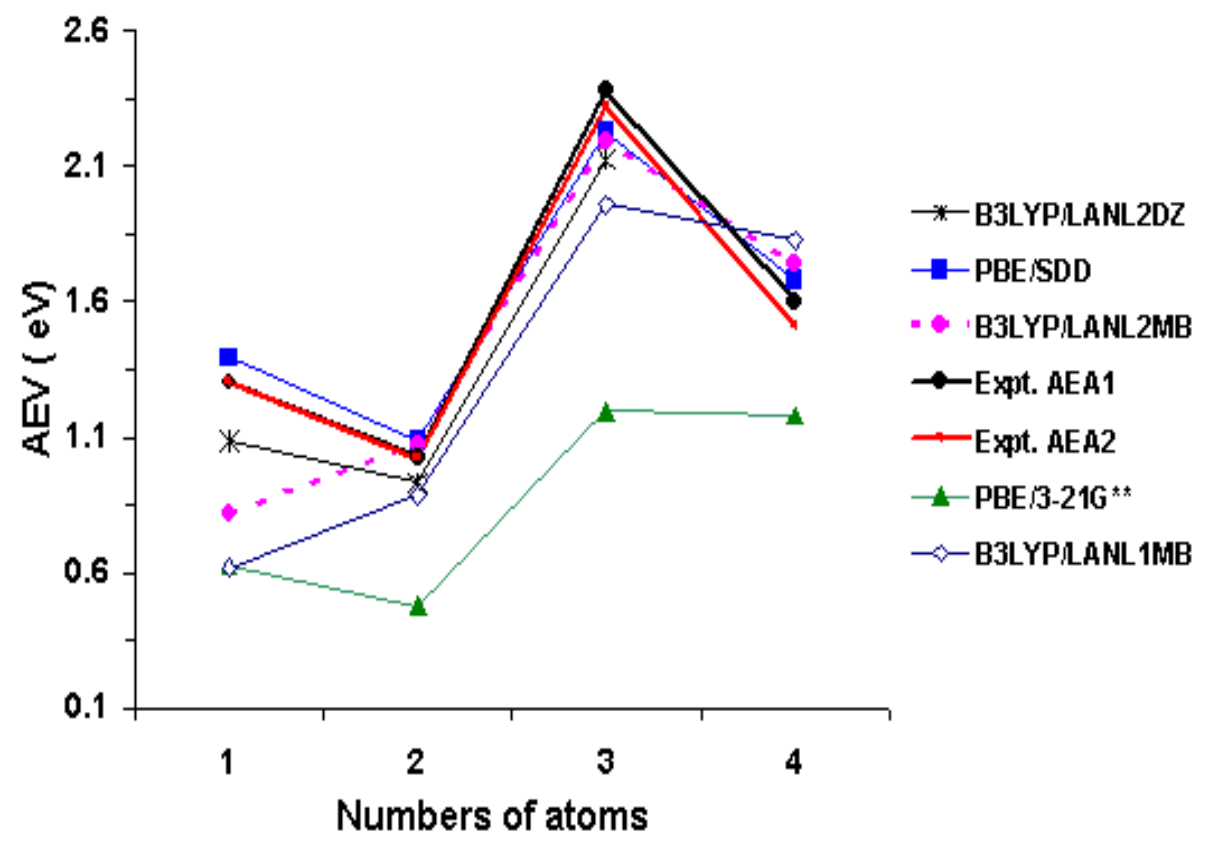

Fig. 43. Vertical electronic afinity for silver cluster employed DFT. Vs. Number of atoms in the cluster. AEA1 experimental [61, 63]. AEA2 experimental[37]

Introducted the electronic correlation effects with PBE functional and the relativistic effects SDD has been obtained, for the adiabatic electronic affinitie, value very nears of the experimental data reported in [62-64, 48] for $A g_{n}(\mathrm{n}=1-4)$ (see Fig. 42 and 43). With the electronic correlation effects $\mathrm{PBE} / 3-21 \mathrm{G}^{* *}$, the values are very distant of the experimental data, see Fig. 42 and 43.

With B3LYP functional and LANL2DZ has been obtained good results for the adiabatic electronic affinity, Fig. 42 and 43.

The results obtained in this work for the silver cluster optimized with MP2/LANL2DZ concured with the results in the literature employed MP2 and LANLEDZ [65].

If compared the results obtained with DFT/PBE/SDD, DFT/PBE/3-21G** and HF/LANL2DZ the relativistic effects only, are not much influenced in adiabatic electronic affinity and neither the electronic correlation effects with PBE functional.

Analyzed the date obtained with DFT/B3LYP/LANL2DZ and DFT/PBE/SDD I see litlle variation.

\subsection{Vertical and Adiabatic Ionization Potential}

The adiabatic ionization potential are defined how the energy of transitionin origin beteen the basal state of cation and the basal state of neutral with $I=E_{n}^{+}-E_{n}$., where $E_{n}^{+}$is the total energie of species of cations after optimization the structure and $E_{n}$ is the total species neutral optimizated [25].

Tendency of the clusters optained with HF, see Fig. 45 and 46 is do not equal with the experimental date $[66,18]$. The values of ionization potential are extensive comparable with electronic affinity $[27,67]$. 
With LANL1MB and LANL2MB change of the dates for dimer and trimer clusters are the same.

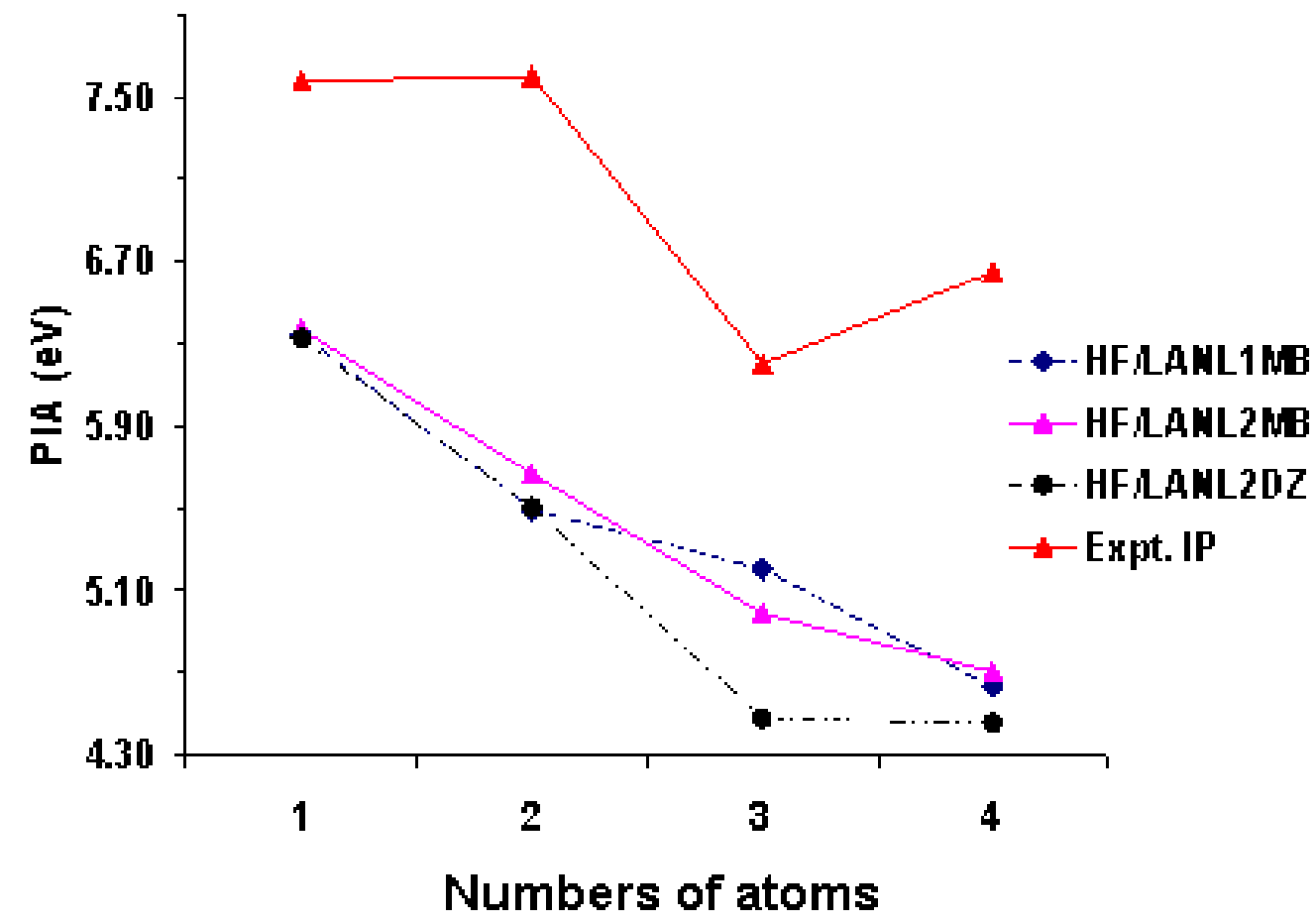

Fig. 44. Adiabatic electronic potential for silver cluster employed HF. Vs. Number of atoms in the cluster:Experimental potential of ionization[6, 46]

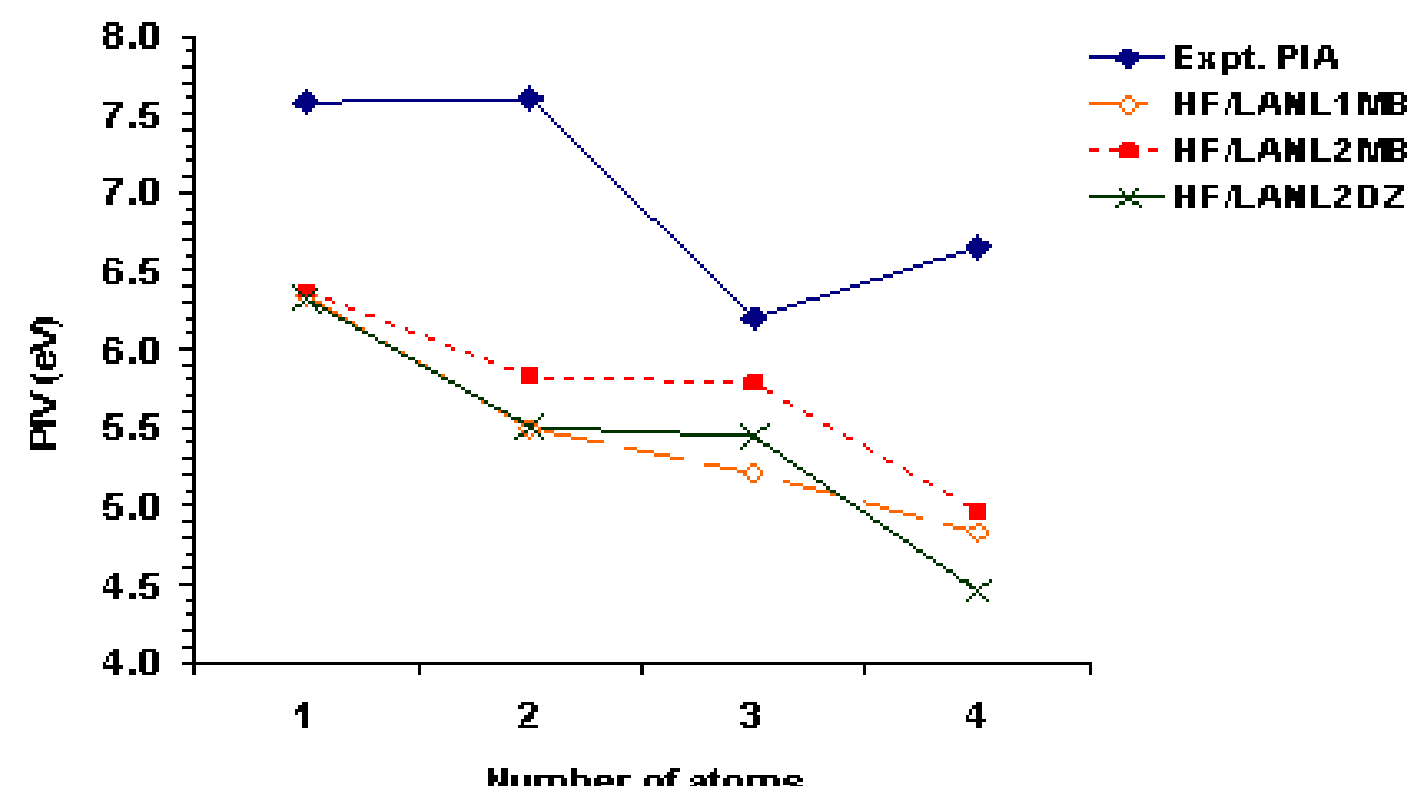

Fig. 45. Vertical ionization potential for silver cluster employed HF. Vs. Number of atoms in the cluster.Experimental potential of ionization[6, 46]

With see the Fig 48 has been optained good results when are employed B3LYP/LANL2MB. The diference with experimental data are de $0.03 \mathrm{eV}$ for one atom, two atoms and three atoms and, $0.14 \mathrm{eV}$ for fourth atoms clusters.

When are employed B3LYP/LANL2DZ level of theorie are obtained $7.75 \mathrm{eV}$ estimated with $7.57 \mathrm{eV}$, the experimental data for one atom, $7.72 \mathrm{eV}$ compared with 7.6 $\mathrm{eV}$, the experimental data for two atoms, see Fig.48.

The better result are optained with B3LYP and are near of the jellium model (SJBM). Are better PBE/SDD who PBE/3-

\section{$21 \mathrm{G}^{* *}$}

The electron is ionizated of the feeble biding orbital, see Fig 6 and7. The IP for three atoms are less who foro over.

\section{Frecuencies}

How see in Table 1 the values better near of the experimental data for two atoms anion and catión has been obtained employed PBE/SDD. For neutral dimer employed PBE $/ 3-21 \mathrm{G}^{* *}$ are optained $199.08 \mathrm{~cm}^{-1}$ forehead to 192.4 (5), 
the experimental data (see Table 1) continued for the DFT/PBE/SDD level.

Tabla 1. The armonic frecuencia (in $\mathrm{cm}^{-1}$ ) of the mas stable silver clusters.

\begin{tabular}{|c|c|c|c|c|c|}
\hline $\mathbf{A g}_{4}$ & $\mathrm{Ag}_{4}^{-}$ & $\overline{\mathrm{Ag}_{4}^{+}}$ & $\mathbf{A g}_{3}$ & $\mathrm{Ag}_{3}^{-}$ & $\mathrm{Ag}_{3}^{+}$ \\
\hline & & & $161.1(157.9)^{\mathrm{a}}$ & & \\
\hline $34.16^{\mathrm{b}}$ & $71.57^{b}$ & $15.56^{\mathrm{b}}$ & $13.25^{\mathrm{b}}$ & $28.44^{\mathrm{b}}$ & $110.80^{\mathrm{b}}$ \\
\hline $71.58^{\mathrm{b}}$ & $134.57^{\mathrm{b}}$ & $71.32^{\mathrm{b}}$ & $112.71^{\mathrm{b}}$ & $89.96^{\mathrm{b}}$ & $110.89^{b}$ \\
\hline $76.25^{\mathrm{b}}$ & $166.53^{\mathrm{b}}$ & $84.80^{\mathrm{b}}$ & $159.16^{\mathrm{b}}$ & $156.12^{\mathrm{b}}$ & $167.53^{\mathrm{b}}$ \\
\hline $100.30^{\mathrm{b}}$ & & $87.45^{\mathrm{b}}$ & & & \\
\hline $152.35^{\mathrm{b}}$ & & $127.64^{\mathrm{b}}$ & & & \\
\hline $174.99^{b}$ & & $160.11^{\mathrm{b}}$ & & & \\
\hline $38.44^{\mathrm{c}}$ & $15.23^{\mathrm{c}}$ & $18.44^{\mathrm{c}}$ & $11.79^{c}$ & $34.94^{c}$ & $117.87^{\mathrm{c}}$ \\
\hline $82.89^{c}$ & $22.95^{\mathrm{c}}$ & $82.03^{\mathrm{c}}$ & $126.41^{\mathrm{c}}$ & $107.76^{\mathrm{c}}$ & $118.49^{c}$ \\
\hline $186.73^{\mathrm{c}}$ & $28.65^{c}$ & $94.76^{\mathrm{c}}$ & $171.33^{\mathrm{c}}$ & $178.23^{\mathrm{c}}$ & $174.66^{\mathrm{c}}$ \\
\hline $92.77^{\mathrm{c}}$ & $78.31^{\mathrm{c}}$ & $103.86^{\mathrm{c}}$ & & & \\
\hline $164.57^{\mathrm{c}}$ & $144.02^{\mathrm{c}}$ & $146.94^{\mathrm{c}}$ & & & \\
\hline \multirow[t]{7}{*}{$186.73^{\mathrm{c}}$} & $178.47^{\mathrm{c}}$ & $169.87^{\mathrm{c}}$ & & & \\
\hline & & $14.87^{\mathrm{d}}$ & $87.72^{\mathrm{d}}$ & $42.97^{\mathrm{d}}$ & $118.71^{\mathrm{d}}$ \\
\hline & & $91.02^{\mathrm{d}}$ & $114.58^{\mathrm{d}}$ & $128.23^{\mathrm{d}}$ & $118.71^{\mathrm{d}}$ \\
\hline & & $97.31^{\mathrm{d}}$ & $197.20^{\mathrm{d}}$ & $196.12^{\mathrm{d}}$ & $184.06^{\mathrm{d}}$ \\
\hline & & $115.05^{\mathrm{d}}$ & $5.63^{\mathrm{e}}$ & & \\
\hline & & $158.53^{\mathrm{d}}$ & $80.55^{\mathrm{e}}$ & & \\
\hline & & $178.05^{\mathrm{d}}$ & $91.70^{\mathrm{e}}$ & & \\
\hline
\end{tabular}

${ }^{a}$ experimental data $[29]^{b}$ the date of the present worck with DFT/B3LYP/LANL2DZ level of theorie

${ }^{c}$ the date of present worck with DFT/PBE/SDD the date of the present worck with DFT/PBE/3-21G** level of theorie

${ }^{e}$ the date of presen worck withl HF/LANL2DZ

Tabla 2. The frecuencies obtained for the silver cluster in gas phase.

\begin{tabular}{lccc}
\hline Métodos & $\mathbf{A g}_{2}$ & $\mathrm{Ag}_{2}$ & $\mathrm{Ag}_{2}^{+}$ \\
\hline Experimental[20,21] & $192.4(5)^{\mathrm{a}, \mathrm{b}}$ & $145.0^{\mathrm{c}}$ & $135.8^{\mathrm{c}}$ \\
DFT/PBE/3-21G** & 199.07 & 165.96 & 141.13 \\
DFT/PBE/SDD & 185.94 & 134.22 & 131.58 \\
DFT/B3LYP/LANL2DZ & 177.06 & 125.61 & 124.16 \\
DFT/B3LYP/LANL2MB & 146.45 & 125.61 & 124.16 \\
HF/3-21G** & 141.12 & 97.26 & 94.48 \\
HF/LANL2DZ & 149.32 & 88.59 & 91.30 \\
\hline
\end{tabular}

a experimental [28]

${ }^{\mathrm{b}}$ experimental [29]

${ }^{c}$ experimental [30]

In the Table 3 are see the anion dimer optained with PBE/SDD and HF/LANL2DZ

Tabla 3. The frecuencies obtained for the silver cluster in acuos solution with the PCM ( $\varepsilon=78.39)$ model.

\begin{tabular}{lccc}
\hline MéTodos solvatación $(\boldsymbol{\varepsilon}=\mathbf{7 8 . 3 9})$ & $\mathbf{A g}_{2}$ & $\mathrm{Ag}_{2}^{-}$ & $\mathrm{Ag}_{2}^{+}$ \\
\hline DFT/PBE/3-21G** & 188.15 & 120.1191 & 157.79 \\
DFT/PBE/SDD & 175.53 & -53.28 & 138.12 \\
DFT/B3LYP/LANL2DZ & 162.00 & 94.47 & 133.35 \\
DFT/B3LYP/LANL2MB & 146.45 & 94.47 & 133.35 \\
HF/3-21G** & 111.75 & 46.23 & 95.51 \\
HF/LANL2DZ & 115.76 & -17.85 & 85.39 \\
\hline
\end{tabular}

\section{Hardness}

Are calculated hardness with the objective see the reactivity of the cluster taken in account the relativistic effects and electronic correlation and see what silver cluster digest how soft acid $r$ soft base $u$ hard acid or hard base. The value of hardnees ar in Fig. 49 and 50. And are employed $\eta=$ (I-A)/2.
With the HF I observ who the more reactive are the structure with for atoms.

When I empolyed LANL1MB the $1.64 \mathrm{eV}$ are obtained, for the LANL2DZ level the value are $1.70 \mathrm{eV}$, see Fig.19.

To be observed who the reactivity increment with el namber of atoms in the cluster and not exist the variation pair unequal. The $\mathrm{Ag}_{3}$ optimised with HF/LANL2DZ is near of the experimental hardness. 
See de hardness experimental in Fig. 49 and 50 [62, 64 68, 66, 18].



Fig. 46. Adiabatic electronic afinity for silver cluster employed DFT. Vs. Number of atoms in the cluster.. AEA1 experimental [61, 63]. AEA2 experimental [37]

In the Fig.50 I see the best reactive are tthree atoms and for atoms.

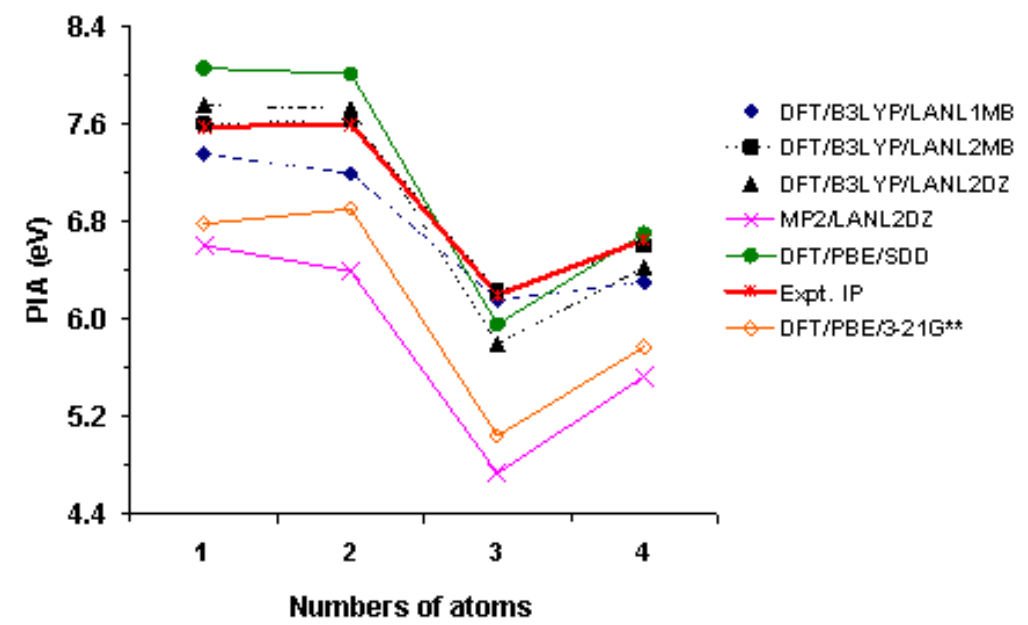

Fig.47. Adiabatic ionization potential for silver cluster employed DFT. Vs. Number of atoms in the cluster.Experimental potential of ionization[6, 46]

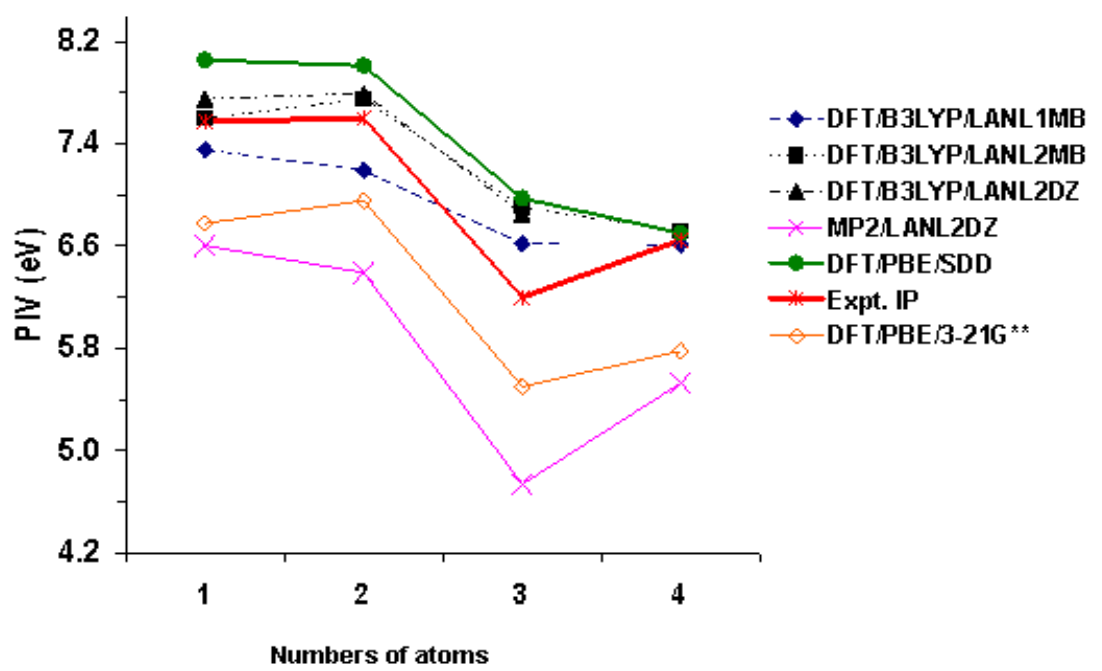

Fig. 48. Vertical ionization potential for silver cluster employed DFT. Vs. Number of atoms in the cluster.Experimental potential of ionization [6, 46] 


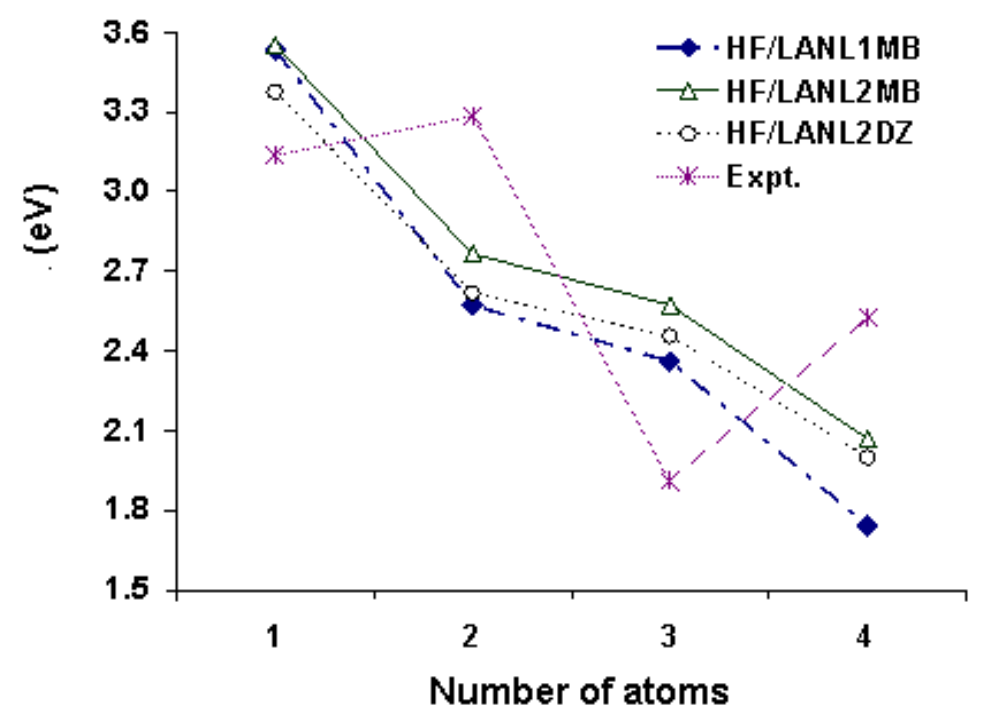

Fig. 49. Hardness ( $\boldsymbol{\eta}$ ) for the silver clusters employed HF. Vs. Number of the atoms in the cluster.

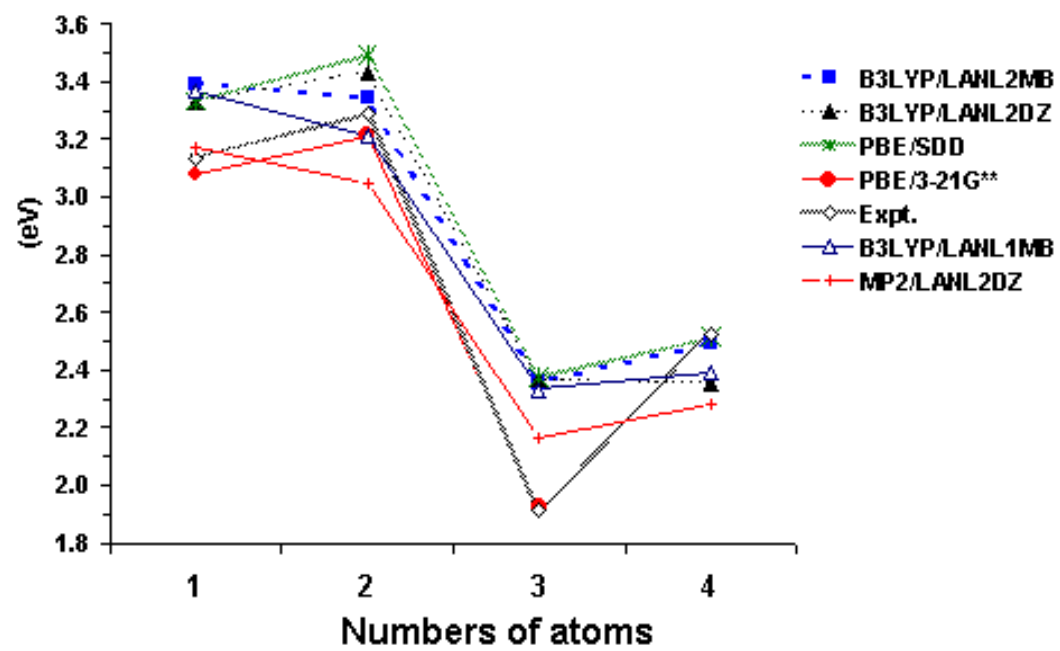

Fig. 50. Hardness ( $\boldsymbol{\eta}$ ) for the silver clusters employed DFT. Vs. Number of the atoms in the cluster.

Inthe Table 4 and 5 are the value obtained for the difference HOMO-LUMO (gap) for the clusters employed the Klopman theorie.[68].

Are calculated the HOMO-LUMO with:

$$
\text { HOMO-LUMO }=\left(-\mathrm{E}_{\mathrm{HOMO}}\right)-\left(-\mathrm{E}_{\mathrm{LUMO}}\right)
$$

$\mathrm{E}_{\mathrm{HOMO}}=$ the energie of the orbital high occupied
$\mathrm{E}_{\mathrm{LUMO}}=$ the energie of the orbital low occupied.

The value optained in the gas phase are smaller in 0.2-0.3 $\mathrm{eV}$ nearly with respect of the values in aqueous solution, it who oppose who the solvent make that increase the difference HOMO-LUMO.

With HF/LANLEDZ and HF/3-21G* has been obtained less values of gap with 2.521 and $2.021 \mathrm{eV}$ who in gas phase.

Tabla 4. The values of HOMO, LUMO, and gap for the Ag2 clusters in the gas phase.

\begin{tabular}{|c|c|c|c|}
\hline Method & HOMO (eV) & LUMO (eV) & HOMO-LUMO gap (eV) \\
\hline $\mathrm{DFT} / \mathrm{PBE} / 3-21 \mathrm{G}^{* *}$ & -4.290 & -2.379 & 1.911 \\
\hline DFT/PBE//SDD & -5.262 & -3.235 & 2.027 \\
\hline DFT/B3LYP/LANL2DZ & -5.558 & -2.653 & 2.905 \\
\hline DFT/B3LYP/LANL2MB & -5.559 & -2.845 & 2.714 \\
\hline $\mathrm{HF} / 3-21 \mathrm{G}^{* *}$ & -5.392 & 0.253 & 5.139 \\
\hline HF/LANL2DZ & -5.949 & 0.030 & 5.979 \\
\hline
\end{tabular}


Tabla 5. Values of HOMO, LUMO, and gap for the $\mathrm{Ag}_{2}$ clusters obtained in acuos solution employed PCM model with $\varepsilon=78.39$.

\begin{tabular}{lccc}
\hline SOLVATATION $(\boldsymbol{\varepsilon}=\mathbf{7 8 . 3 9})$ & & & \\
\hline Method & HOMO $(\mathbf{e V})$ & LUMO $(\mathbf{e V})$ & HOMO-LUMO gap $(\mathbf{e V})$ \\
\hline DFT/PBE/3-21G** & -3.230 & -1.021 & 2.209 \\
DFT/PBE//SDD & -4.230 & -1.836 & 2.394 \\
DFT/B3LYP/LANL2DZ & -4.572 & -1.276 & 3.295 \\
DFT/B3LYP/LANL2MB & -4.170 & -1.259 & 2.912 \\
HF/3-21G** & -4.300 & 1.682 & 2.618 \\
HF/LANL2DZ & -5.175 & 1.217 & 3.958 \\
\hline
\end{tabular}

Tabla 6. Values of HOMO, LUMO, and gap for the $\mathrm{Ag}_{3}$ clusters obtained in the gas phase.

\begin{tabular}{lccc}
\hline Method & HOMO $(\mathbf{e V})$ & LUMO $(\mathbf{e V})$ & HOMO-LUMO gap $(\mathbf{e V})$ \\
\hline DFT/PBE/3-21G** & -4.129 & -2.814 & 1.314 \\
DFT/PBE//SDD & -5.136 & -3.600 & 1.536 \\
DFT/B3LYP/LANL2DZ & -5.419 & -3.039 & 2.380 \\
DFT/B3LYP/LANL2MB & -5.411 & -3.164 & 2.247 \\
HF/3-21G** & -5.045 & -3.164 & 1.881 \\
HF/LANL2DZ & -5.354 & -3.164 & 2.190 \\
\hline
\end{tabular}

\section{Conclusions}

Introducing the electronic correlation effects without reelativistic effect with the $\mathrm{PBE}$ functional and the $3-21 \mathrm{G}^{* *}$ has been optained the best results of $2.53 \AA$, compared with PBE/SDD with wich has been obtained 2,57 $\AA$ for neutral dimer.

Of where has been concluid who the electronic correlation effects modify more in the distance beteen the clusters that the relativist effects.

The anion clusters almost all case are the lineal form how the most stable, the cation of equilateral triangle and isosceles.

At make additions un electron the clusters of two and for atoms optimized with HF are better stable what of the three atoms, in barter for the cation cluster at remove un electron perform who the cluster with pair number are less stable who the cluster with unequal number.

Not be able make the same for the clusters optimized introduce the electronic correlation, in this case the charge are uniformly disposed beteen the three atoms in the lineal form.

Introduced the density funtional are incluyed in B3LYP the LDA funtional it who make what the result obtained with DFT/B3LYP/LANL2MB it be more near of the experimental data, respecting the model of jellium layer.

In this case I observed who the relativistic effects not influence in the proprieties how the elctronic affinity and ionization potential. Compared $\mathrm{PBE} / 3-21 \mathrm{G}^{* *}$ and $\mathrm{PBE} / \mathrm{SDD}$ the electronic correlation are complementary wiht the relativistic effect. The MP2 are improper with the electronic afinity and ionization potential.

The results obtaine with $\mathrm{PBE} / 3-21 \mathrm{G}^{* *}$ and $\mathrm{PBE} / \mathrm{SDD}$ for the frecuencies are the near of the experimentals date.
The values of the hardness are near of the experimentals data have been obtained for PBE/3-21G** following for $\mathrm{PBE} / \mathrm{SDD}$, whey the electronic correletion are important compiting with the tow effects the relativistic with electronic correlation.

\section{References}

[1] Huda M.N.; Ray A. K.; Phys. Rev. A, 2003, 67, 013210.

[2] Fournier R., J. Chem. Phys., 2001, 2165.

[3] Wnag B.; Chen X.; Wang J.; Zhao J., Surface Review and Letters, 2004, 11,15.

[4] Monti O.L.A.; Fourkas, J.T.;Nesbitt D.J., J. Phys. Chem. B, 2004, 108, 1604.

[5] Zhang L.;Yuu J.C., Ho Yin Yip; Li Q.; Kwong K. W.; Xu A. Wu; Wong Po K.; Langmuir, 2003, 19, 10372.

[6] Yuranava T.; Rincon A.G.; Bozzi A.G.; Parra S.; Pulgarin C.; Albers P.; Kiwi J., J. Photochem, Photobiol.A., 2003, 161, 27.

[7] Empedocles; Neuhauser R.; Shimizu K.; Bawendi M.G., Adv. Mater., 1999, 11, 1243.

[8] Link S.; El-Sayed, Int Rev. Phys. Chem., 2000, 19, 409.

[9] Andersen P.C.; Rowlen K.L., Appl. Spectroscop., 2002, 56, $124 \mathrm{~A}$.

[10] Henglein A., Chem. Rev. 1989, 89, 1861.

[11] Kim S.H.; Ribeiro G.M.; Ohlberg D.A.A.; Williams R.S., Heath J.R., J.Phys. Chem., 1999, 103, 10341.

[12] Zuhuang J., Bactericidal nanosilver cloth and its making proces and use. Patent number CN 1387700, 2003. 
[13] Chen C.M.S., Process for preparing antibacterial antimildew polyacrylic fibers and its filter net for air condiyioner. Patent number CN 1355335, 2003.

[14] Lee H.J.; Yeo .S.Y.; Jeong, J. Mat. Sci., 2003,38, 2199.

[15] Balasubramanian K., J. Phys. Chem., 1989, 93, 6585.

[16] Ichihara K.T.; Fujita Y.;Matsuo T., Sakuray T.; Matsuda H., Int J. Mass, Spectrom. In. Proc., 1985, 67, 229; ibid. 1986, 74, 33.

[17] Handschuh H.; Cha C.Y.; Bechthold P.S.; Ganteför, Eberhatdt W., J. Chem. Phys., 1995, 102, 6406.

[18] Haslett T.L.; Bosnick K.A.; Fedrigo S.; Moskovits M., J. Chem. 1999, 11, 14, 6456,

[19] Wedum E.E.; Grant E.R.; Cheng P.Y.; Willy K.F.; Duncan M.A., J. Chem. Phys. Lett., 1991, 100, 6312.

[20] Félix C.; Sieber C.; Harbich W.; Buttet J.; Rabin I.; Schultze W. Ertl, Chem. Phys. Lett., 1999, 313, 195.

[21] Howard J.A.; Sutcliffe R.; Mile B., Surf. Sci., 1985, 156, 214.

[22] Haslett T.L.; Bosnick K.A.; Moskovits M., J. Chem. Phys., $1998,108,3453$.

[23] Rabin I.; Jackschath C.; Schultze W., Z. Phys. D, 1991, 19, 153. Jackschath, Rabin I.; Schultze W., ibid, 1992, 22, 517.

[24] Allameddin G.; Hunter J., Cameron D.; Kappes M.M., Chem. Phys. Lett, 1992, 192, 122.

[25] Ho J.; Ervin K.M.; Lineberger, J. Chem. Phys. 1990, 93, 6987.

[26] Leopold, D.G.; Ho J.; Leneberger W.C., J. Chem. Phys., 1987, $86,1715$.

[27] Taylor K.J., Pettiette-Hall C.L.; Cheshnovsky O.; Smalley, J. Chem. Phys., 1992, 96, 3319.

[28] Handuschu H.; Chaa C. Y.; Bechtold P.S., Ganteför G.; Eberhatdt, J. Chem. Phys., 1995, 102, 6406.

[29] Okazaki T.; Saito Y., Kasuya A., Nishina Y., J. Chem. Phys., 1996, 104, 812

[30] Tiggesbäumeker T.; Köller L., Meiwes-Broer K.; Liebesch A., Phys. Rev. A, 1993, 48, 1749.

[31] Minemoto, Iseda M., Kondow T., Eur. Phys. J.D., 1999, 9, 163.

[32] Bonačič Koutecký V.; Češpiva; Fantucci P.; Pittner J.; Koutecký J., J. Chem. Phys., 1994, 100, 1.

[33] Santamaria R.; Kaplan I. G.; Novaro O.; Chem. Phys. Letters, 1994, 218, 395.

[34] Liu Z.F.; Yim W.L.; Tse J.S.; Hafner J., Eur. Phys. J.D., 2000, 10,105 .

[35] Zhao J.; Luo Y.; Wang Eur. Phys., J. D, 2001, 14, 309.

[36] Legge Sue F., Nyberg Graeme L., Peel Barrie J., J. Phys. Chem A, 2001, 105, 7905.

[37] Weis P.; Bierweiler T.; Gilb S.; Kappes M.M., Chem Phys. Lett., 2002, 355, 355.

[38] Mitrić, Hartmann M.; Stanca B.; Bonačič Koutecký V.; Fantucci, J. Phys. Chem., 2001, A 105, 8892.

[39] Poteau R.; Heully J.L.; Spiegelman F.; Z. Phys. D, 1997, 49,
479.

[40] Tian Z.M., Tian Y.; Wei W.M.; He T.J.; Chen D.M.; Liu F.C., Chem. Phis. Lett, 2006, 420, 450.

[41] Wedum E.E., Grant E.R., Chang P.Y.; Willey K.F.; Duncan M.A., J. Chem. Phys., 1994,100.

[42] Cheng P.Y.; Duncan M.A., Chem Phys. Lett., 1988, 152, 341.

[43] Ellis M.; Robles E.S.J., Millar I.A., Chem Phys. Letter.,1993, 201132.

[44] Bonačič Koutecký V.; Veyeret V.; Mitric R., J. Chem. Phys., 2001, 115, 10450.

[45] Hay P.J.; Wadt W.R., J. Chem. Phys., 1985, 82, 284.

[46] Hay P.J.; Wadt W.R., J. Chem. Phys., 1985, 82, 299.

[47] Boo D. Wan; Ozaki Y.; Andersen L. H.; Lineberger W.C., J. Phys. Chem A., 1997, 101, 6688.

[48] Ho J.; Ervin K.M., Lienberger W.C., J. Chem. Phys. 1990, 93, 6987.

[49] Bagatur'yants A.A.; Safanov A.A., Stoll H.; Werner H.J., J. Chem. Phys., 1998, 109, 3096.

[50] Boo Wan D., Ozaki Y., Andersen L. H.; Lineberger W.C., J. Phys. Chem A., 1997, 101, 6688.

[51] Schultze W.; Becker H.U.; Minkwitz R.; Mansel K., Chem. Phys. Letters, 1978, 55, 59.

[52] Moskowits M., DiLella D.P., J. Chem. Phys., 1980, 72, 2267.

[53] Joward J.A.; Preston K.F.; J. Am. Chem. Soc. 1981, 103, 6226.

[54] Kernisant K., Thompson G.A., Lindsay D.M., J. Chem. Phys., $1985,82,4739$.

[55] Morse M.D., Chem. Rev. 1986, 86, 1049.

[56] Bonačić-Koutecký V.; Češpiva L., J. Chem. Phys., 1993, 98, 7981.

[57] Basch H., J. Am. Chem. Soc., 1981, 103, 4657.

[58] Matulis V.E; Ivashkevich O.A.; Gurin V.S, J. Molec. Struct. (Theochem), 2003, 664-665, 291.

[59] Häkkinen H.; Moseler M.; Landman Uzi, Physical Rev. Lett., 2002, 89, 033401-1.

[60] Huda M.N.; Ray A.K., Eur. Phys. J. D, 2003, 22, 217.

[61] Boo Wan D.; Ozaki Y, Andersen L. H.; Lineberger W.C, J. Phys. Chem A., 1997, 101, 6688.

[62] Spasov V.A., Lee T.H. ; Maberry J.P. ; Ervin K.M., J. Chem. Phys., 1999, 110, 5208,

[63] Shi Y., Spasov V.A., Ervin K.M., J. Chem. Phys., 1999, 111, 938.

[64] Moore C.E., Atomic energy levels, NSRDS-NBS Circular No. 467, USGPO, Washington, 1949.

[65] Zuhuang J., Bactericidal nano-silver cloth and its making process and use. Patent number CN 1387700, 2003.

[66] Rabin I., Jackschath C.; Schulze W., Z. Phys. D, 1991, 19, 153,. Jackschath C., Rabin I.; Schulze W., ibid. 1992, 22, 517. 
[67] Ekardt W., Phys. Rev. B, 1984, 29, 1558.

[68] C.E. Moore, Atomic energy levels, NSRDS-NBS Circular No. 467, USGPO, Washington, 1949.
[69] Foresman J.B., Frisch A., Gaussian, Inc. Pittsburgh, PA, 230249, 1996. 